\title{
Le traitement conservateur dans le cadre d'une pubalgie de surcharge chez l'athlète, une revue systématique de revues systématiques
}

\author{
MEGANE BARRAS
}

Etudiante HES - Filière Physiothérapie

JOANNA LUTZ

Etudiante HES - Filière Physiothérapie

Directeur de travail de Bachelor : NICOLAS MATHIEU

TRAVAIL DE BACHELOR

Déposé à Loèche-les-bains (VS-CH) le 9 juin 2017

En vue de l'obtention d'un

Bachelor of sciences HES-SO in Physiotherapy 


\section{Résumé}

\section{Introduction}

La pubalgie de surcharge touche de nombreux athlètes et engendre d'importantes conséquences, tant musculo-squelettiques que financières. De nombreux facteurs de risque ont été identifiés mais cette pathologie reste complexe et il n'existe aucun consensus concernant le traitement conservateur.

L'objectif de cette étude est de répertorier les différents traitements conservateurs dans le cadre de pubalgies de surcharge chez les athlètes et d'en évaluer l'efficacité pour émettre des recommandations thérapeutiques pratiques (GRADE) pour le physiothérapeute.

\section{Méthode}

Cette étude est une revue systématique de revues systématiques (overview) enregistrée auprès de Prospero (CRD42017065268). Nous avons exploré trois bases de données ainsi que la littérature grise à l'aide d'une stratégie de recherche boléenne. La recherche et la sélection des articles ont été réalisées de manière indépendante. La qualité des articles a été analysée de manière individuelle à l'aide de la check-list AMSTAR. Notre travail a été conceptualisé selon les lignes directrices PRISMA et les recommandations formulées selon le système GRADE.

\section{Résultats}

Sur 1852 articles, nous en avons sélectionné trois. L'analyse des données montre que le traitement multimodal est supérieur au traitement actif par l'exercice avec notamment la possibilité de retourner au sport un mois plus tôt. Le traitement actif par l'exercice permet de meilleurs résultats que le traitement passif.

\section{Discussion - Conclusion}

Il ressort de cette étude que les physiothérapeutes devraient appliquer une thérapie multimodale comme prise en charge de leurs athlètes atteints de pubalgie, afin d'améliorer l'efficacité de la prise en charge conservatrice.

Mots-clés : pubalgie / athlètes / traitement conservateur 


\section{Zusammenfassung}

\section{Einleitung}

Viele Athleten leiden wegen Überbelastung an Leistenschmerzen und das hat muskuloskelettale sowie finanzielle Folgen. Viele Risiko-Faktoren wurden identifiziert, aber diese Pathologie bleibt komplex und es existiert zur Zeit noch kein Konsens über die konservative Behandlung.

Das Ziel dieser Studie ist, die verschiedenen konservativen Behandlungen aufzulisten, ihre Wirksamkeit $\mathrm{zu}$ bewerten, um dann praktische therapeutische Empfehlungen (GRADE) für Physiotherapeuten abzugeben.

\section{Methode}

Diese Studie ist eine systematische Review die in Prospero verzeichnet ist (CRD42017065268). Wir haben drei Datenbasen und die graue Literatur erforscht durch eine boolesche Suchstrategie. Die Recherche und die Auswahl der Artikel sind unabhängig realisiert worden. Die Qualität der Artikel ist durch die AMTAR Checkliste analysiert worden. Unsere Arbeit ist nach den PRISMA Leitlinien geschrieben worden und die Empfehlungen nach dem GRADE System formuliert.

\section{Ergebnisse}

Aus den 1852 Artikeln haben wir drei Artikel ausgewählt. Die Datenanalyse zeigt, dass die multimodale Behandlung bessere Resultate erzielt als die einfache aktive Behandlung. Sie ermöglicht dem Sportler auch, einen Monat früher wieder Sport zu treiben. Die aktive Behandlung erlaubt bessere Ergebnisse als die passive Behandlung.

\section{Diskussion - Schlussfolgerung}

Nach der Erforschung der Literatur und der Analyse den systematischen Review, geht hervor, dass Physiotherapeuten für Athleten, die an Leistenschmerzen leiden die multimodale Behandlung als Therapie benutzen sollten, um die Effizienz der konservativen Behandlung zu verbessern.

Stichwörter : Leiste-Schmerz / Athleten/ konservative Behandlung 


\section{Abstract}

\section{Introduction}

Many athletes are affected by exercise-related groin pain and this results in significant musculoskeletal and financial consequences. Numerous risk factors have been identified but this pathology is still complex and there is actually no consensus on the conservative treatment of groin pain.

The purpose of this study is to list all the different conservative treatments of exerciserelated groin pain by athletes, to assess their effectiveness and finally provide practical therapeutic recommendations for the physiotherapists.

\section{Method}

This study is an overview registered with Prospero (CRD42017065268). We searched through three databases and the grey literature using a bolean research strategy. The research and the selection process of the articles were realised independently by the two authors. The quality assessment of the articles was achieved individually using the AMSTAR check-list. Our work has been conceptualized on the basis of the PRISMA guidelines as well as and the recommandations formulated within the GRADE system.

\section{Results}

From the 1852 articles, three have finally been selected. The data analysis shows that multimodal treatment is more efficient than simple exercise therapy, including the possibility to return to play one month earlier. Exercise therapy gives better results than passive treatment.

\section{Discussion - Conclusion}

After exploring the literature and analysing systematic reviews, we concluded that physiotherapists should use multimodal treatment to manage athletes suffering from groin pain in order to improve the conservative treatment.

Keywords : groin pain / athletes / conservative treatment 


\section{Avertissement}

Les prises de position, la rédaction et les conclusions de ce travail n'engagent que la responsabilité de ses auteurs et en aucun cas celle de la Haute Ecole de Santé Valais, du directeur du Travail de Bachelor ou du Jury.

Nous attestons avoir réalisé seules la présente revue sans avoir utilisé d'autre source que celles indiquées dans la méthode (cf. Point 2.2).

Loèche-les-Bains, le 09.06.2017

Mégane Barras et Joanna Lutz 


\section{Remerciements}

Nous souhaitons remercier les personnes suivantes :

Monsieur Nicolas Mathieu, enseignant à la HES-SO Valais-Wallis, filière physiothérapie et directeur de travail de Bachelor, pour son soutien ainsi que ses conseils et ses remarques tout au long de la réalisation de notre revue « panorama ».

Madame Anne-Gabrielle Mittaz, enseignante à la HES-SO Valais-Wallis, filière physiothérapie, pour son aide concernant la méthodologie d'une revue systématique de revues systématiques.

Monsieur Roger Hilfiker, enseignant à la HES-SO Valais-Wallis, filière physiothérapie, pour son aide dans l'utilisation du logiciel RevMan et de l'analyse des statistiques.

Mme P. Barras, M. N. Legris, Mme N. Däpp, Mme K. Schaller et M. M. Stornetta pour la relecture attentive de notre travail.

Mlle K. Gaspoz et Mme S. Chambovey pour la vérification des traductions.

Nos proches et familles respectives, pour leur soutien, leurs encouragements et leur patience. 


\section{Table des matières}

1.1 CONTEXTE GÉNÉRAL

1.2 CONCEPTUALISATION

1.2.1 LA PUBALGIE

1.2.2 FACTEURS DE RISQUE

1.3 TRAITEMENT CONSERVATEUR ET TRAITEMENT CHIRURGICAL 6

1.3.1 LE TRAITEMENT CONSERVATEUR

1.3.2 LE TRAITEMENT CHIRURGICAL

1.5 QUESTION DE RECHERCHE

\subsection{DESIGN}

2.2 BASES DE DONNÉES

2.3 STRATÉGIE DE RECHERCHE

2.4 CRITÈRES D'INCLUSION

2.5 CRITÈRES D'EXCLUSION

2.6 SÉLECTION DES ARTICLES

2.7 EVALUATION DE LA QUALITÉ DES ÉTUDES COLLIGÉES 10

2.8 GESTION DES DONNÉES

2.9 RÉDACTION DE NOTRE ÉTUDE

2.10 FORMULATION DES RECOMMANDATIONS

3.1 RÉSULTATS DE LA RECHERCHE ÉLECTRONIQUE

Etude 1 : thérapie active par l'exercice VS physiothérapie conventionnelle - Hölmich et al. (1999)

Etude 2 : thérapie multimodale VS thérapie active par l'exercice - Weir et al. (2011)

REVUE 2 : MACHOTKA ET AL. (2009)

REVUe 3 : SeRner et Al. (2015) 
8. ANNEXES

ANNEXE I : DIAGNOSTICS DIFFÉRENTIELS 29

ANNEXE II : EXTRACTION DES DONNÉES (ALMEIDA)

30

ANNEXE III : EXTRACTION DES DONNÉES (MACHOTKA)

32

ANNEXE IV : EXTRACTION DES DONNÉES (SERNER)

34

ANNEXE $V$ : QUALITÉ EN FONCTION DES POINTS AMSTAR

36 


\section{Abréviations}

AMSTAR : A Measurement Tool to Assess Systematic Review

CI : Confidence Interval

HAS : Haute Autorité de la Santé

M. : Muscle

MD : Mean Deviation

N. : Nerf

RCT : Randomised controlled trial

RR : Risk Ratio

RS: Revue systématique

RTP : Return to play

SD: Standard deviation

TENS: Transcutaneous Electrical Nerve Stimulator

Th : Thérapie

TM : Thérapie manuelle

TTT : Traitement

VAS : Visual Analog Scale 


\section{Introduction}

\subsection{Contexte général}

Les pubalgies touchent les sportifs amateurs comme professionnels et sont relativement fréquentes puisqu'elles représentent 2 à $5 \%$ des blessures dues au sport et figurent à la troisième place sur la liste des blessures dans le cadre du football (Albers, Zwerver, Diercks, Dekker, \& Van den Akker-Scheek, 2016 ; Bisciotti et al., 2015 ; de Sa et al., 2016 ; Pruvost, 2015 ; Quinn, 2010 ; Sedaghati, Alizadeh, Shirzad, \& Ardjmand, 2013). Certains auteurs affirment qu'environ $10 \%$ des footballeurs souffrent d'une pubalgie (Paajanen, Ristolainen, Turunen, \& Kujala, 2010). L'incidence des blessures au niveau de l'aine est de 0.8/1000 heures de pratique du football (Ekstrand \& Hilding, 1999). En Australie, environ quatorze matchs par année sont manqués dans chaque club suite à cette blessure (Cowan et al., 2004). Cette pathologie représente aussi un enjeu financier; le nombre de matchs manqués sur une saison à cause des pubalgies représente une perte de 1.7 million de dollars australiens (Pizzari, Coburn, \& Crow, 2008). La période de convalescence peut durer plusieurs mois et a un coût important autant pour l'athlète que pour le système de santé (Holmich, Larsen, Krogsgaard, \& Gluud, 2010).

Les sports dans lesquels se retrouve la plus forte prévalence sont les sports acycliques tels que le football, le hockey, le tennis, le rugby, la course de longue distance, le basketball, la danse, etc. (Almeida, Silva, Andriolo, Atallah, \& Peccin, 2013 ; Bisciotti et al., 2015 ; Elattar, Choi, Dills, \& Busconi, 2016 ; Pruvost, 2015 ; Puig, Trouve, \& Savalli, 2004 ; Quinn, 2010). Les femmes sont cependant bien moins touchées que les athlètes de sexe masculin avec une incidence de 2.1/1000 heures de football dans les clubs masculins contre 0.6/1000 chez les footballeuses (Puig et al., 2004 ; Waldén, Hägglund, \& Ekstrand, 2015). Les patients souffrant de pubalgie ont généralement entre 20 et 50 ans (Yuill, Pajaczkowski, \& Howitt, 2012).

Holmich et al. (2010) ont démontré qu'un patient ayant déjà eu une blessure à l'aine double ses risques d'en avoir une deuxième. De plus, le fait de jouer à un plus haut niveau triple ce risque (Holmich et al., 2010). 


\subsection{Conceptualisation}

\subsubsection{La pubalgie}

Les pubalgies sont caractérisées par une douleur au niveau inguino-pubien, souvent aggravées par la toux, les éternuements, les coups de pied, les rotations, etc. et elles regroupent plusieurs diagnostics établis en fonction des structures atteintes qui peuvent être nombreuses (Bisciotti et al., 2015 ; Elattar et al., 2016 ; Pruvost, 2015 ; Puig et al., 2004).

Il est difficile de donner une seule définition de la pubalgie ; cela est dû à sa complexité, relevant en partie du grand nombre de structures anatomiques pouvant être en cause ou des différents mécanismes de lésions possibles. On trouve également dans la pratique une opposition non encore résolue entre les spécialistes américains, pour qui la base de la symptomatologie se trouve au niveau de l'aine (Groin pain) et les européens qui la voient plutôt dans la zone du pubis (Meyers et al., 2008 ; Pruvost, 2015 ; Puig et al., 2004 ; Adam Weir et al., 2015). Généralement, la pubalgie peut être classifiée en deux groupes :

1. La pubalgie micro-traumatique ou traumatique qui découle d'un traumatisme de la symphyse pubienne. Elle peut survenir suite à une chute sur un pied qui provoque le soulèvement d'une des deux branches pubiennes et du même coup un mouvement de cisaille au niveau du pubis, étirant les ligaments pubiens et pouvant même bloquer la partie supérieure de la branche pubienne. Une autre possibilité de lésion des ligaments et des insertions musculaires au niveau du pubis peut être le résultat d'une tension soudaine des adducteurs.

2. La pubalgie chronique ou de fatigue, due aux tensions importantes que les muscles imposent au bassin et pouvant toucher différentes zones. Dans le cas de la pubalgie haute, l'inflammation est localisée au niveau de l'insertion du grand droit de l'abdomen (le grand droit et les obliques tirant vers crânial). Pour la pubalgie basse, ce sont les adducteurs, muscles très utilisés chez les athlètes, qui sont atteints (ils tirent le bassin vers caudal) (de Larra y Arnaiz, 2011).

Les causes ont été classées en grandes catégories: orthopédique, neurologique, oncologique, rhumatologique, urologique, gastro-intestinale, etc. (Adam Weir et al., 2015).

Bouvard et ses collègues (2004) ont défini quatre catégories cliniques: l'ostéoarthropathie pubienne (au niveau de la symphyse), les douleurs du canal inguinal, les 
tendinopathies à l'insertion du grand droit et les tendinopathies des adducteurs (Bouvard, Dorochenko, Lanusse, \& Duraffour, 2004).

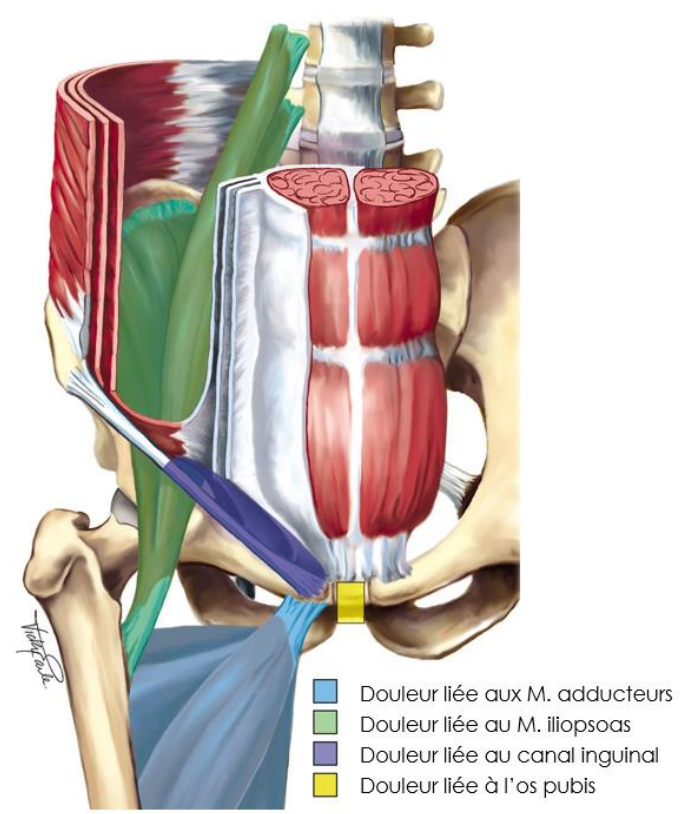

Figure 1 : Illustration de Cornford (Langhout, Tak \& Glasgow, 2016)

Il est fréquent de trouver plusieurs atteintes pour un même cas de pubalgie (Bouvard et al., 2004 ; Caudill, Nyland, Smith, Yerasimides, \& Lach, 2008 ; Munegato et al., 2015). Falvey et al. (2009) parlent du triangle de l'aine, appelé aussi triangle de Scarpa, triangle fémoral ou triangle inguinal, pour différencier les diverses pathologies : des fascias, neurovasculaires et musculosquelettiques (Falvey, Franklyn-Miller, \& McCrory, 2009 ; Leitner \& Sherwood, 1983 ; Micheletti et al., 1998 ; Radojević, 1962). Les repères anatomiques sont l'épine iliaque antérieure supérieure, le tubercule pubien et le mid-thigh point 3G, un point à mi-cuisse (Falvey et al., 2009; Khan \& Al Tunaiji, 2016). Il est délimité par les structures suivantes: au niveau supérieur le ligament inguinal, médialement le muscle long adducteur et latéralement le muscle sartorius (Micheletti et al., 1998). Ils définissent les causes par rapport au bord atteint (Khan \& Al Tunaiji, 2016). Les causes les plus fréquentes de pubalgies sont la déchirure des muscles adducteurs, la hernie du sportif, l'osteitis pubis ainsi que l'impingement fémoro-acétabulaire. Cependant il existe plusieurs diagnostics différentiels comme le démontre le tableau de Khan (Khan \& Al Tunaiji, 2016) et l'annexe 1 [Annexe I : diagnostics différentiels]. 


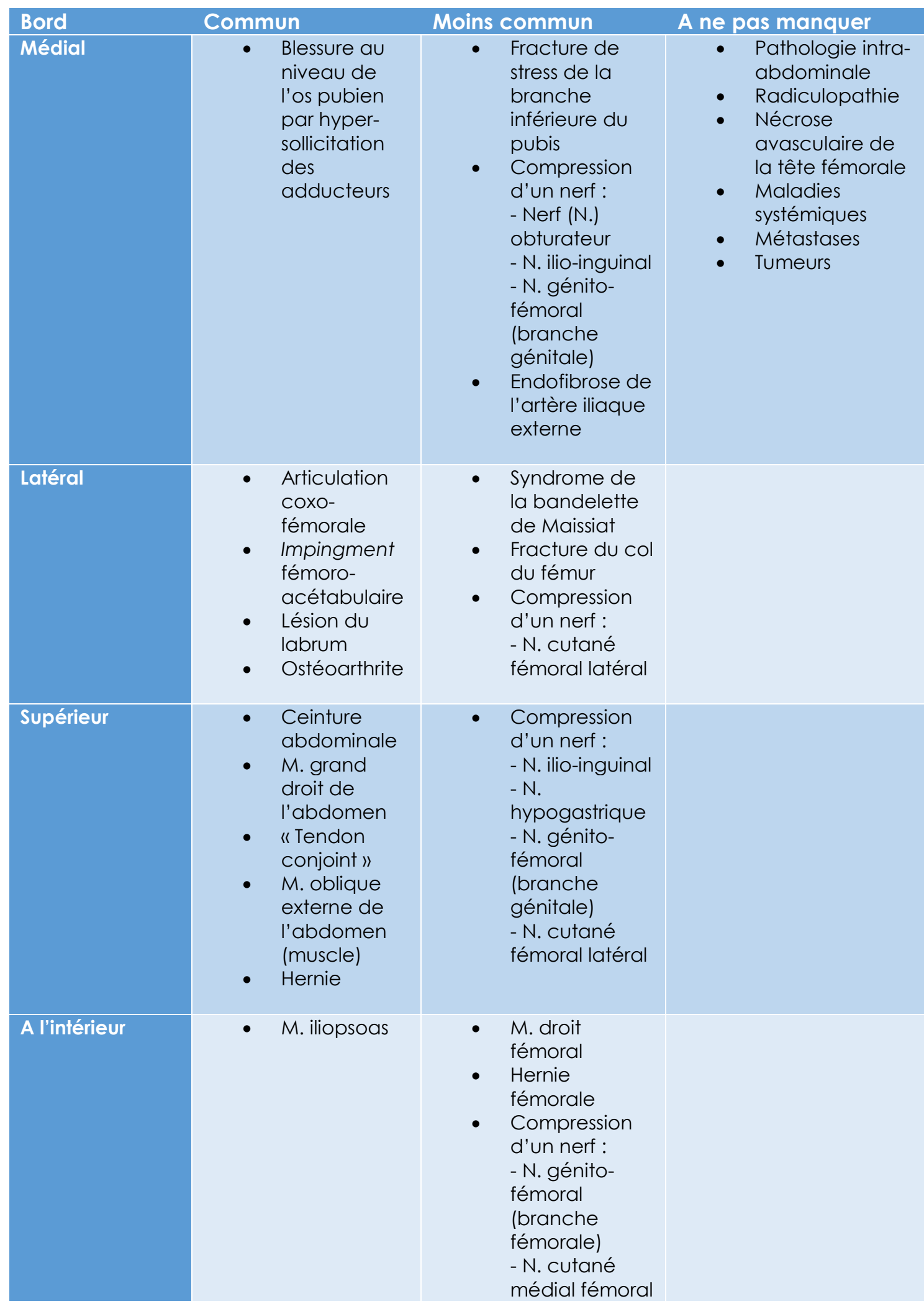

Tableau 1 : Etiologies des douleurs chroniques à l'aine chez les athlètes, tiré de (Khan \& Al Tunaiji, 2016) et traduit par Barras et Lutz (2017)

Certains auteurs évoquent aussi la hernie de Spieghel (hernie ventrale latérale) (D. W. Larson \& Farley, 2002). Aucun consensus n'a encore été trouvé concernant cette 
pathologie, mis à part celui sur la terminologie (Yuill et al., 2012). De plus, un « expert round » réunissant 24 experts internationaux a défini une classification de la pubalgie en trois grandes catégories selon les répercussions sur le sport et la vie quotidienne (Adam Weir et al., 2015) :

- Les entités cliniques causes de pubalgies, soit : les adducteurs, l'iliopsoas, la zone inguinale et la zone du pubis

- Les pubalgies d'origine coxofémorale

- Les autres causes touchant les athlètes

Concernant les tendinopathies, il existe plusieurs classifications; l'une des plus complètes est celle de Leadbetter (1992) qui se catégorise en quatre stades (Leadbetter, 1992):

\begin{tabular}{|c|c|c|c|c|}
\hline & $\begin{array}{l}\text { Expression de la } \\
\text { douleur }\end{array}$ & Clinique & Evoluant depuis & $\begin{array}{l}\text { Anatomo- } \\
\text { pathologie }\end{array}$ \\
\hline Stade 1 & $\begin{array}{l}\text { Douleur après le } \\
\text { sport, } \\
\text { disparaissant au } \\
\text { repos en } \\
\text { quelques heures }\end{array}$ & $\begin{array}{l}\text { Pas de signe } \\
\text { clinique } \\
\text { particulier }\end{array}$ & $\begin{array}{l}\text { Moins de } 2 \\
\text { semaines }\end{array}$ & $\begin{array}{l}\text { Lésions } \\
\text { microscopiques } \\
\text { et réversibles }\end{array}$ \\
\hline Stade 2 & $\begin{array}{l}\text { Douleur pendant } \\
\text { et après le sport } \\
\text { ne disparaissant } \\
\text { pas ou peu au } \\
\text { repos }\end{array}$ & $\begin{array}{l}\text { Douleur localisée } \\
\text { Peu ou pas de } \\
\text { signes } \\
\text { inflammatoires }\end{array}$ & $\begin{array}{l}\text { Entre } 2 \text { et } 6 \\
\text { semaines }\end{array}$ & $\begin{array}{l}\text { Lésions } \\
\text { microscopiques } \\
\text { et réversibles }\end{array}$ \\
\hline Stade 3 & $\begin{array}{l}\text { Douleur } \\
\text { persistant } \\
\text { plusieurs jours } \\
\text { après l'arrêt de } \\
\text { l'activité, } \\
\text { réapparition } \\
\text { rapide à la } \\
\text { reprise } \\
\text { Limitations } \\
\text { fonctionnelles } \\
\text { nettes }\end{array}$ & $\begin{array}{l}\text { Présence de } \\
\text { signes } \\
\text { inflammatoires }\end{array}$ & $\begin{array}{l}\text { Supérieure à } 6 \\
\text { semaines }\end{array}$ & $\begin{array}{l}\text { Apparition de } \\
\text { lésions } \\
\text { macroscopiques } \\
\text { et irréversibles }\end{array}$ \\
\hline Stade 4 & $\begin{array}{l}\text { Douleur } \\
\text { permanente } \\
\text { empêchant } \\
\text { toute activité } \\
\text { sportive et } \\
\text { gênant les } \\
\text { activités } \\
\text { quotidiennes } \\
\text { courantes }\end{array}$ & $\begin{array}{l}\text { Signes } \\
\text { inflammatoires } \\
\text { importants }\end{array}$ & & $\begin{array}{l}\text { Dans la majorité } \\
\text { des cas, } \\
\text { présence de } \\
\text { lésions } \\
\text { macroscopiques } \\
\text { et irréversibles }\end{array}$ \\
\hline
\end{tabular}


Les pubalgies sont généralement dues à un surmenage lors d'activités spécifiques telles que la pratique de la course à pied, les changements de direction soudains, les demi-tours explosifs, les rotations, les changements de rythme ou encore les tirs dans un ballon. (Bisciotti et al., 2015 ; Drew, 2015 ; Elattar et al., 2016 ; Grairi, 2011 ; Puig et al., 2004 ; Trofa, Mayeux, Parisien, Ahmad, \& Lynch, 2017).

\subsubsection{Facteurs de risque}

Des facteurs de risque ont été identifiés et peuvent être séparés en facteurs intrinsèques tels que, entre autres, une faiblesse des abdominaux ou des muscles de la ceinture pelvienne, des adducteurs beaucoup plus faibles que les abducteurs (norme ratio $=0.64$ (Sugimoto, Mattacola, Mullineaux, Palmer, \& Hewett, 2014)), un manque de mobilité des hanches, un manque de souplesse au niveau de l'aine, l'âge, une instabilité articulaire, le morphotype, des prédispositions génétiques, etc. et en facteurs extrinsèques tels qu'une mauvaise posture, un mauvais échauffement, un surmenage et la fatigue liés à un excès d'activité physique, du matériel mal adapté, une blessure récurrente, le genre, etc. (Bisciotti et al., 2015 ; Elattar et al., 2016 ; Grairi, 2011 ; Holmich et al., 2010 ; Ishøi et al., 2016 ; McAleer, Gille, Bark, \& Riepenhof, 2015 ; Munegato et al., 2015 ; Quinn, 2010 ; Sedaghati et al., 2013).

\subsection{Traitement conservateur et traitement chirurgical}

\subsubsection{Le traitement conservateur}

Dans la littérature parcourue, la prise en charge thérapeutique conservatrice des pathologies de surcharge n'est pas souvent décrite précisément. Néanmoins nous avons pu exploiter certaines recommandations thérapeutiques. Dans notre étude, nous différencions la pathologie de surcharge de la pathologie structurelle (herniation, hernie, ...).

Jusqu'à peu, le traitement non opératoire était considéré comme étant sans succès (Hackney, 1993 ; Joesting, 2002 ; LeBlanc \& LeBlanc, 2003 ; Morelli \& Smith, 2001). Cependant, depuis quelques années, plusieurs programmes de soins conservateurs ont été développés en se fondant sur «l'evidence based medicine» (Caudill et al., 2008). Plusieurs traitements (TTT) sont proposés pour une prise en charge optimale. L'objectif est de retrouver la mobilité et la force ainsi que les capacités fonctionnelles et sensorimotrices et ce en commençant par traiter un des symptômes les plus importants : 
la douleur. (Ellsworth, Zoland, \& Tyler, 2014). Certains auteurs proposent de débuter dans tous les cas par une période de repos (Almeida et al., 2013). En physiothérapie, il existe plusieurs options de traitements pour les pubalgies : l'électrothérapie (ultrasons, Transcutaneous Electrical Nerve Stimulator (TENS), laser, ...), la thermothérapie, la cryothérapie, les massages (techniques d'assouplissement cutané, pressions localisées, frictions, techniques de «crochetage », étirements), la thérapie manuelle (TM), le travail de la mobilité articulaire et de la souplesse musculaire (étirements, postures), ou encore le renforcement des muscles stabilisateurs du pelvis et de la hanche (Almeida et al., 2013 ; Grairi, 2011).

Plusieurs études séparent la rééducation en trois parties : la prise en charge de la douleur, suivie du traitement des facteurs de risque influençables (par le travail de la force et de la stabilité) et finalement une progression fonctionnelle comprenant la reprogrammation neuro-sensori-motrice, ainsi que le travail des éléments spécifiques au sport du patient avec comme objectif final le retour au sport (Grairi, 2011 ; St-Onge, MacIntyre, \& Galea, 2015).

\subsubsection{Le traitement chirurgical}

Le traitement chirurgical quant à lui était vu comme la meilleure option envisageable dans le cadre d'une atteinte structurelle de type hernie du sportif. Cette position a cependant été revue et, selon plusieurs auteurs, il y a en réalité trois indications pour l'opération : un traitement conservateur insatisfaisant, une sensation de déchirure ressentie par le patient et la situation particulière de l'athlète professionnel qui n'a pas le temps pour un long traitement conservateur (Farber \& Wilckens, 2007 ; Kachingwe \& Grech, 2008 ; C. M. Larson \& Lohnes, 2002).

\subsection{Objectif}

En explorant la littérature, nous avons constaté qu'il n'y avait pas de consensus établi quant à la prise en charge conservatrice de la pubalgie. Notre premier objectif est de répertorier les différents traitements conservateurs dans le cadre des pubalgies de surcharge chez les athlètes et d'en évaluer l'efficacité pour émettre des recommandations thérapeutiques pratiques (GRADE) pour le physiothérapeute. 


\subsection{Question de recherche}

«Quels sont les traitements conservateurs les plus efficaces lors de pubalgies de surcharge chez les athlètes afin d'émettre des recommandations thérapeutiques ?» $\mathrm{Au}$ début de notre recherche, notre hypothèse personnelle était que le traitement conservateur le plus efficace consiste en une période de repos et d'antalgie suivi de thérapie (th) active comprenant renforcement musculaire et stabilisation sensori-motrice.

\section{Méthode}

\subsection{Design}

Nous avons effectué une revue systématique de revues systématiques, appelée aussi revue «parachute», revue «panorama» ou «overview» en anglais, selon les recommandations de Smith et al. (Smith, Devane, Begley, \& Clarke, 2011).

\subsection{Bases de données}

Notre recherche d'articles pertinents sur le sujet a exploré les bases de données électroniques suivantes : PubMed, Embase et Cochrane. Nous avons également utilisé Google Scholar et Google Medical pour rechercher d'éventuels articles de la littérature grise n'ayant pas été publiés sur les moteurs de recherche cités ci-dessus.

\subsection{Stratégie de recherche}

Notre stratégie de recherche s'est formalisée avec la méthode boléenne, en utilisant des mots clés et des Mesh Terms classés en plusieurs catégories. La première catégorie est celle de la pathologie («groin pain», «pubalgia», «osteitis pubis», «gracilis syndrome », «hockey groin», «footballers groin», «conjoined tendon lesions », «posterior abdominal wall defficience », «pubalgia », «athletic pubalgia », « adductor strain », «adductor tendinopathy »). La deuxième est celle de la population («athletes », «sportsmen »). La troisième est celle de l'intervention («conservative treatment», «physical therapy», «stretching», «massage», «strengthening », «functional training », «manual therapy», «manipulation», «myofascia», «rehabilitation 
exercises », «plyometric exercise», «therapeutic modalities», «taping »). La quatrième catégorie était celle des critères d'exclusion («operation», «surgery», «diagnosis », « hernia »).

Afin d'élargir nos recherches, nous n'avons pas inclus d'issues dans les termes de nos recherches. Nous avons cependant déterminé les indicateurs les plus pertinents pour un sportif qui sont, selon nous: la douleur, la qualité de vie, la force musculaire des abdominaux et des membres inférieurs et le retour au sport. Nous les avons utilisés lors de la lecture du texte intégral pour sélectionner les articles.

\subsection{Critères d'inclusion}

Les articles inclus dans notre étude devaient intégrer les critères suivants : être publiés après 2006, traiter des pubalgies de surcharge chez les athlètes (population), parler de traitement conservateur (intervention) et être des revues systématiques ou méta-analyses.

\subsection{Critères d'exclusion}

Les articles d'un autre design que les revues systématiques ou méta-analyses, publiés avant 2006, traitant de pubalgies traumatiques, de traitement chirurgical ou de traitement conservateur après une opération, touchant des non-athlètes, ont été éliminés. Ont été également écartés, les articles écrits dans une langue autre que l'anglais, l'allemand, le français ou l'italien. Il en a été de même pour les articles qui ne répondaient pas aux critères de qualité de la grille AMSTAR (cf. pt. 2.7).

\subsection{Sélection des articles}

Les deux investigatrices ont recherché et sélectionné les articles de manière indépendante puis mis en commun leurs sélections et en cas de conflit, discuté de leurs choix. Un consensus a été atteint dans tous les cas.

Pour la première étape, les doublons ainsi que la langue des articles ont été repérés et le cas échéant éliminés. Nous avons ensuite sélectionné les articles d'après leur titre. Le quatrième tri s'est fait d'après l'abstract. Lors de la cinquième étape, nous avons procédé à la lecture intégrale des articles sélectionnés. Durant ces deux étapes, nous avons éliminé les articles ne correspondant pas au PIOTS (population, intervention, outcome, time, 
study design). Pour terminer, nous avons supprimé encore deux articles d'après leur qualité.

\subsection{Evaluation de la qualité des études colligées}

Afin de définir la qualité de nos études au niveau méthodologique, la grille d'évaluation spécifique aux revues systématiques AMSTAR (A Measurement Tool to Assess Systematic Review) a été utilisée. Cet outil, mis au point par Shea et al. (2007), repose sur 11 questions portant notamment sur le processus de sélection des études, les caractéristiques des études retenues, l'évaluation de la qualité des études ou encore sur la méthodologie employée pour l'analyse statistique (Shea et al., 2007). Il est possible de faire l'évaluation en ligne sur le site amstar.ca. Les articles ont été évalués de manière indépendante puis mis en commun. Etant donné l'importante variation de la qualité des publications, il est essentiel d'utiliser une méthode d'évaluation rigoureuse, afin d'éviter d'éventuels biais liés à la renommée d'un auteur, d'une institution ou d'un journal (Smith et al., 2011).

Les discordances ont été réglées par une discussion qui a abouti dans tous les cas.

Afin de garantir la qualité de notre étude, nous avons décidé, de manière arbitraire, de ne sélectionner que les articles atteignant 8 points sur 11. La Société Française de Santé Publique appuie ce type de prise de décision : «Le processus de réalisation d'une revue systématique applique une succession de prises de décisions, parfois arbitraires ou subjectives »(Zaugg, Savoldelli, Sabatier, \& Durieux, 2014). La synthèse de l'évaluation de la qualité des études a été formalisée avec le logiciel RevMan.

\subsection{Gestion des données}

Les données pertinentes des études ont été extraites et enregistrées dans un tableau formalisé à cet effet [Annexe II : Extraction des données (Almeida)], [Annexe III : Extraction des données (Machotka)], [Annexe IV : Extraction des données (Serner)]. Pour les données manquantes, nous avions envisagé de prendre contact avec les auteurs, ce qui a été fait. Après avoir terminé l'extraction des données, une mise en commun a été effectuée. Selon les données obtenues et leur type, nous avons dû faire des compromis (par exemple dans le choix des outcomes que nous avions choisis au départ). 


\subsection{Rédaction de notre étude}

Pour la rédaction de notre travail, nous avons utilisé les recommandations internationales PRISMA (Moher, Liberati, Tetzlaff, \& Altman, 2009). Le 28 septembre 2016, nous avons enregistré notre projet de revue dans le site dédié aux revues systématiques Prospero et à cette date, il n'y avait aucun autre travail en cours concernant le traitement conservateur des pubalgies qui soit officiellement enregistré (conservative treatment of groin pain). Nous avons confirmé l'enregistrement de notre étude le 4 mai 2017 sous le numéro d'enregistrement CRD42017065268.

$\mathrm{Au}$ vu du peu de données quantitatives contenues dans les revues sélectionnées, nous avons effectué une analyse narrative.

Une fois la rédaction de notre étude terminée, nous l'avons soumise à Urkund pour la vérification de nos sources.

\subsection{Formulation des recommandations}

Pour formuler nos recommandations, nous avons utilisé le système GRADE (Guyatt, Santesso, \& Akl, 2004). Il a été adopté par l'Organisation Mondiale de la Santé et préconisé par la Haute Autorité de la Santé (Dhénain, 2013).

\section{Résultats}

\subsection{Résultats de la recherche électronique}

Notre équation de recherche a permis d'identifier 1828 articles dans les bases de données citées précédemment et 24 articles issus de la littérature grise. In fine, la sélection rigoureuse des articles pertinents a permis d'inclure dans cette revue «parachute » trois revues systématiques (fig. 2 flow chart). 


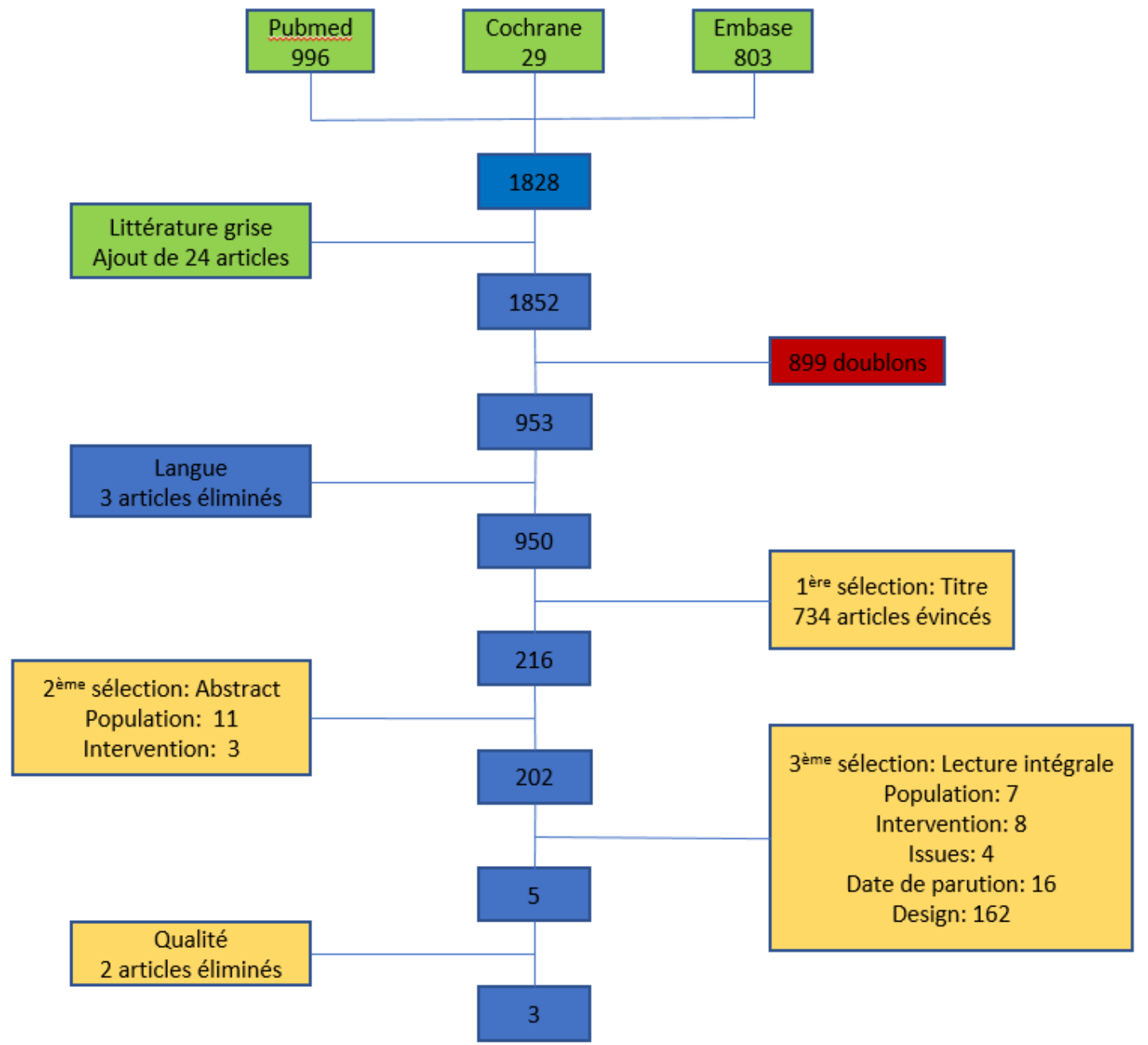

Figure 2 : Flow chart de la sélection des articles de Barras et Lutz (2017)

\subsection{Evaluation de la qualité}

La cotation de la qualité des articles que nous avons analysés à l'aide de l'échelle AMSTAR figure dans le tableau ci-dessous (Tableau 3 : Évaluation de la qualité des articles selon AMSTAR). Pour l'item 5 de la check-list AMSTAR, nous avons contacté les auteurs afin d'obtenir des précisions. Etant donné qu'aucune réponse ne nous est parvenue, nous avons considéré que les noms des études exclues devaient être explicités dans l'article afin de pouvoir lui accorder le point. 


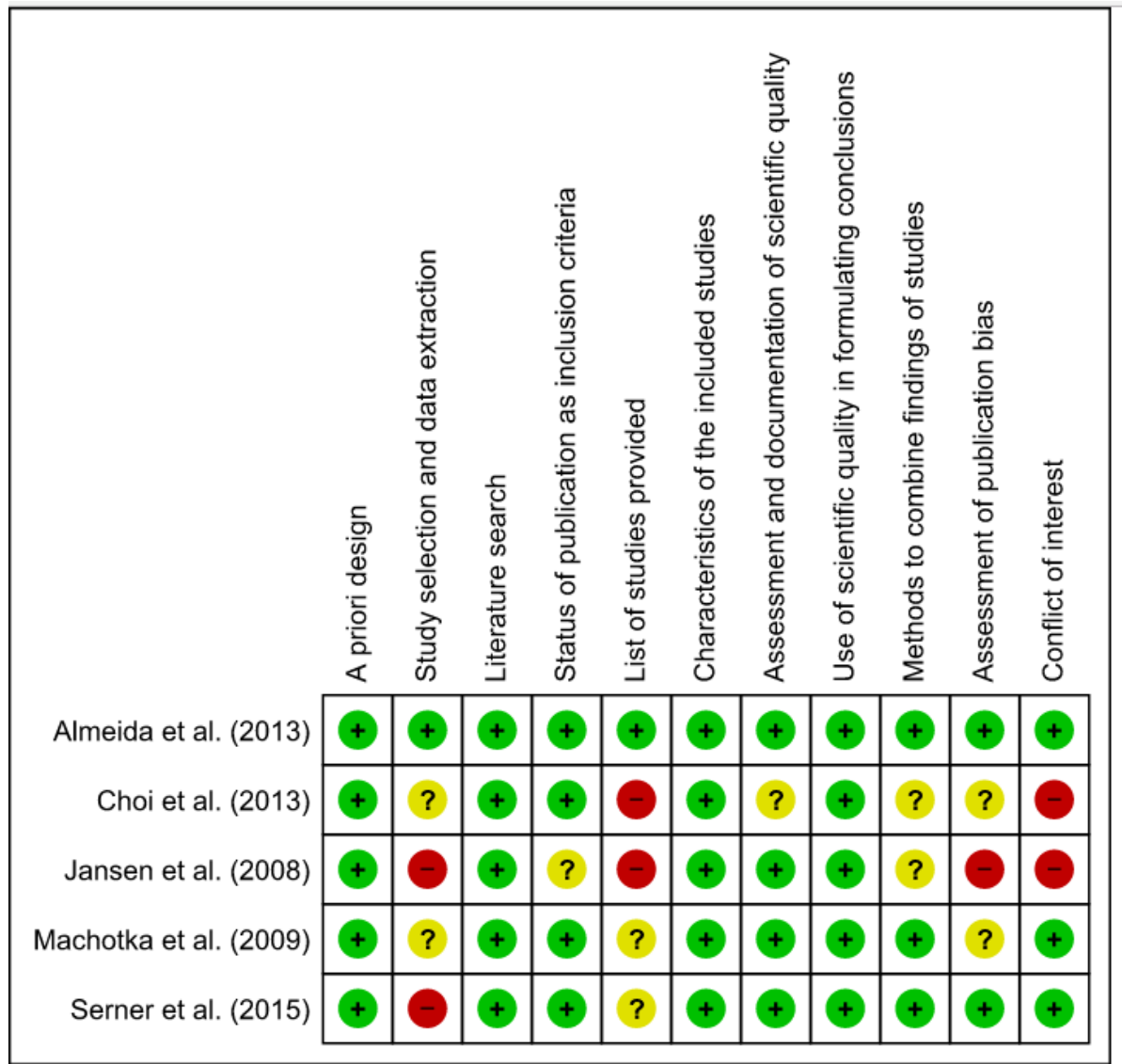

Figure 3 : Evaluation de la qualité selon Amstar (Barras et Lutz, 2017)

La revue systématique d'Almeida et al. (2013) a été cotée à 11/11, Choi et al. (2013) à 5/11, Jansen et al. (2008) à 5/11, Machotka et al. (2009) à 8/11 et Serner et al. (2015) à 9/11. Les articles qui n'ont pas atteint 8 points (cf. 2.7) ont été écartés de notre étude afin de ne garder que les trois articles de bonne qualité. Nous n'avons pas trouvé de littérature sur l'analyse de la qualité et ceci était donc une décision arbitraire dans le but de garantir la qualité de notre revue [Annexe V : Qualité en fonction des points AMSTAR]. 


\subsection{Description des études incluses}

\begin{tabular}{|c|c|c|c|}
\hline Auteurs & $\begin{array}{c}\text { Nbre d'études / } \\
\text { population } \\
\text { (nbre) }\end{array}$ & Intervention & Issues observées \\
\hline $\begin{array}{l}\text { Almeida et al. } \\
2013\end{array}$ & $2 / 122$ & $\begin{array}{l}\text { Thérapie active par } \\
\text { l'exercice VS physiothérapie } \\
\text { conventionnelle (Hölmich, } \\
\text { 1999) } \\
\text { Th. multimodale VS Th. } \\
\text { active par l'exercice (Weir, } \\
2011 \text { ) }\end{array}$ & $\begin{array}{ll}\text { - } & \text { Douleur } \\
\text { - } & \text { Fonction } \\
\text { - } & \text { Succès du TTT } \\
& \text { du paluation subjectivent } \\
\text { - } & \text { Retour au sport (RTP) et } \\
& \text { aux activités normales } \\
\text { - } & \text { Evénements } \\
& \text { indésirables }\end{array}$ \\
\hline $\begin{array}{l}\text { Machotka et } \\
\text { al. } 2009\end{array}$ & $5 / 135$ & $\begin{array}{l}\text { - Thérapie active par } \\
\text { l'exercice }\end{array}$ & $\begin{array}{ll}\text { - } & \text { Douleur } \\
\text { - } & \text { Tests de longueur M. } \\
\text { add } \\
\text { - } \\
\text { Tests de force des M. } \\
\text { add } \\
\text { - } R T P\end{array}$ \\
\hline $\begin{array}{l}\text { Serner et al. } \\
2009\end{array}$ & 72 / 219 & $\begin{array}{l}\text { - } \quad \text { TTT conservateur VS } \\
\text { chirurgical } \\
\text { - } \quad \text { TIT multimodal (ac TM) VS Th. } \\
\text { active simple VS Th. passive }\end{array}$ & $\begin{array}{ll}\text { - } & \text { \% de patients sans } \\
& \text { symptômes } \\
\text { - } & \text { \% patients RTP } \\
\text { - } & \text { Satisfaction du patient } \\
\text { - } & \text { Douleur } \\
\text { - } & \text { Score fonctionnel } \\
\text { - } & \text { Succès du TTT }\end{array}$ \\
\hline
\end{tabular}

Tableau 3 : Description des études incluses (Barras et Lutz, 2017)

\section{Revue 1 : Almeida et al. (2013)}

La revue systématique avait comme objectif «d'évaluer les effets des traitements conservateurs pour les pubalgies de surcharge d'origine musculo-tendineuse, ligamenteuses et osseuse » (Almeida et al., 2013).

La population étudiée était les athlètes atteints de pubalgie de surcharge causée par une dysfonction au niveau de la symphyse, une enthésopathie ou une blessure aux adducteurs. L'évaluation était faite à court terme (4-6 semaines après la fin du traitement), à moyen terme (après 12-16 semaines) et à long terme (1 an après) si possible.

Nous avons pris le parti d'extraire les données obtenues par Intention to treat (sensitivity analysis) car elles prennent en compte tous les patients répartis dans les groupes au moment de la randomisation. Les avantages sont de maintenir la comparabilité entre les deux groupes et de réduire le risque de biais et de faux positifs dans les résultats (Kleist, 2009).

La revue systématique d'Almeida et al. (2013) reprend les études de Hölmich (1999) qui compare la thérapie active par l'exercice avec la physiothérapie conventionnelle (passive) et de Weir (2011) qui confronte la thérapie multimodale avec la thérapie active par 
l'exercice (Almeida et al., 2013 ; Per Hölmich, Nyvold, \& Larsen, 2011 ; A. Weir et al., 2011).

\section{Etude 1: thérapie active par l'exercice VS physiothérapie conventionnelle - Hölmich et al. (1999)}

Dans l'étude de Hölmich (1999), l'issue primaire est le succès du traitement qu'il définit comme une absence de douleur au repos, à la palpation et durant l'activité physique (Per Hölmich et al., 1999). Sur un suivi de 16 semaines, il démontre d'une manière statistiquement significative que la thérapie active par l'exercice (groupe d'intervention) est supérieure à la physiothérapie conventionnelle (25/34 (74\%) versus 10/34 (29\%); risk ratio $(\mathrm{RR}) 2.50,95 \%$ confidence interval $(\mathrm{CI}) 1.43$ to $4.37, \mathrm{P}=0.001)$. Sur un suivi de 8 à 12 ans, la différence n'est plus significative (RR $1.17,95 \%$ CI 0.77 to $1.76, \mathrm{P}=0.46)$.

Concernant les issues secondaires, Hölmich (1999) prouve d'une manière statistiquement significative qu'il y a un plus grand pourcentage d'athlètes retournant au sport au même niveau et sans douleur dans le groupe d'intervention (RR 5.75, 95\% CI 2.23 to $14.86, \mathrm{P}$ $=0.0003)$ (Per Hölmich et al., 1999). Les résultats de l'évaluation subjective globale par le patient sont également en faveur de la thérapie active mais les différences ne sont pas statistiquement significatives pour le suivi à 16 semaines (RR 1.07, 95\% CI 0.86 to 1.34 , $\mathrm{P}=0.53)$ ni à long terme $(\mathrm{RR} 1.25,95 \% \mathrm{CI} 0.79$ to $1.97, \mathrm{P}=0.34)$. Le nombre de traitements était quasiment égal entre le groupe d'intervention (15 fois) et le groupe contrôle (14 fois).

\section{Conclusion de l'étude 1 :}

La thérapie active par l'exercice est supérieure à la physiothérapie conventionnelle en termes de succès du traitement à 16 semaines et du nombre d'athlètes de retour au sport.

\section{Etude 2 : thérapie multimodale VS thérapie active par l'exercice - Weir et al. (2011)}

Dans l'étude de Weir et al. (2011), il y a deux issues primaires (A. Weir et al., 2011). L'auteur n'observe pas de différence statistiquement significative pour le succès du traitement (RR $1.01,95 \%$ CI 0.6 to $1.71, \mathrm{P}=0.96)$. Concernant la douleur maximale durant le sport (visual analog scale (VAS) 0-100), bien que les chiffres soient en faveur 
de la thérapie active par l'exercice par rapport au traitement multimodal, ils ne sont pas statistiquement significatifs (mean deviation (MD) $15.10,95 \% \mathrm{CI}-7.29$ to $37.49, \mathrm{P}=$ $0.19)$.

L'issue secondaire est le retour au sport au même niveau et sans douleur. Il n'y a pas de différences statistiquement significatives entre les deux groupes par rapport au nombre d'athlètes de retour au jeu ( $\mathrm{RR} 0.93,95 \% \mathrm{CI} 0.53$ to $1.66, \mathrm{P}=0.82)$. Le groupe d'intervention (multimodal) est retourné au sport en moyenne 4.5 semaines avant le groupe contrôle, ce qui est statistiquement significatif (12.6 weeks versus 17.3 weeks ; MD-4.5 weeks, $95 \%$ CI -8.60 to $-0.40, \mathrm{P}=0.03)$.

\section{Conclusion de l'étude 2 :}

Le traitement multimodal permet un retour au sport plus rapide que la thérapie active simple.

\section{Revue 2 : Machotka et al. (2009)}

La revue systématique avait deux objectifs : évaluer l'évidence des données actuelles sur l'efficacité de la thérapie active par l'exercice pour les pubalgies chez les athlètes et identifier les facteurs clés de la thérapie active par l'exercice pour les traiter.

Elle étudiait les athlètes atteints de pubalgie et comprenait tous les types d'études.

L'étude de Machotka et al. (2009) traite de la thérapie active par l'exercice (Machotka, Kumar, \& Perraton, 2009).

Le retour au sport est favorable selon toutes les études incluses (P. Hölmich et al., 1999 ; McCarthy \& Vicenzino, 2003 ; Rodriguez, Miguel, \& Lima, 2001 ; Verrall, Slavotinek, Fon, \& Barnes, 2007 ; Wollin \& Lovell, 2006). Les meilleurs résultats sont obtenus lorsque la thérapie est administrée en petits groupes d'une à quatre personnes avec comme co-intervention le jogging, la course ou le vélo. Selon deux études menées dans le milieu $\mathrm{du}$ football et du football australien, le traitement permet une rémission des symptômes (Rodriguez et al., 2001 ; Verrall et al., 2007). En termes de retour au sport sans douleur, Hölmich et ses collègues (1999) ont observé de bons résultats à partir de 90 minutes de renforcement des muscles abdominaux et des muscles de la hanche, trois fois par semaine durant 8 à 12 semaines (P. Hölmich et al., 1999). Cependant, il est le seul auteur inclus dans cette revue systématique qui aie utilisé un programme prédéfini et donné 
suffisamment de détails sur les modalités de traitement; il y a donc une absence d'évidence concernant l'intensité et la fréquence les plus efficaces.

Pour les mêmes raisons, il est impossible de définir précisément les muscles à cibler dans la prise en charge mais il est néanmoins important de noter que dans toutes les études incluses, le focus est toujours mis sur les muscles abdominaux et ceux de la hanche. Toutes les études incluses donnent des résultats positifs à court terme. En revanche, seuls deux auteurs avancent des effets positifs sur le long terme. Le premier n'observe aucune rechute subjective des symptômes à un an (Wollin \& Lovell, 2006). Le second a rapporté un retour au sport de $100 \%$ des athlètes à deux ans mais il modère ce résultat en rappelant les enjeux pour ces patients (Verrall et al., 2007).

Selon l'évidence à disposition, le renforcement musculaire est une composante importante du traitement conservateur. Dans quatre études utilisant le renforcement, la progression se fait à l'aide d'exercices isocinétiques à travers plusieurs degrés de mobilité (P. Hölmich et al., 1999 ; McCarthy \& Vicenzino, 2003 ; Rodriguez et al., 2001 ; Wollin \& Lovell, 2006). Trois d'entre elles commencent par des contractions statiques isométriques puis progressent vers des positions fonctionnelles à travers différentes plages de mouvements (P. Hölmich et al., 1999 ; McCarthy \& Vicenzino, 2003 ; Wollin $\&$ Lovell, 2006). Toutes les études soulignent le besoin de progression au cours de la période d'intervention, ce qui est cliniquement significatif car cela prend en compte les gains obtenus durant le traitement (P. Hölmich et al., 1999 ; McCarthy \& Vicenzino, 2003 ; Rodriguez et al., 2001 ; Verrall et al., 2007 ; Wollin \& Lovell, 2006).

Dans cette revue systématique, la durée d'intervention varie de 3.8 à 16 semaines, ce qui peut être expliqué par le grand nombre de degrés de pubalgie inclus.

\section{Conclusion de cette revue :}

La thérapie active par l'exercice est efficace en termes de retour au sport et de rémission des symptômes. Le renforcement musculaire et la progression sont des éléments essentiels de la prise en charge.

\section{Revue 3 : Serner et al. (2015)}

La revue systématique avait comme objectif de parcourir la littérature afin d'identifier l'efficacité des traitements des pubalgies chez les athlètes. 
La population étudiée était les athlètes atteints de pubalgie. La revue systématique comprend les études randomisées contrôlées, des séries de cas, des études de cas et des études contrôlées de type prospectif ou rétrospectif.

L'étude de Serner regroupe les traitements chirurgicaux et conservateurs dans le cadre des douleurs à l'aine de longue durée liées aux adducteurs. Nous avons procédé à une analyse sensitive en excluant les études sur la chirurgie et en nous concentrant uniquement sur les comparaisons entre les différents traitements conservateurs tels que la thérapie multimodale et la thérapie active par l'exercice. (Serner et al., 2015).

Il y a une évidence modérée que la thérapie active par l'exercice (renforcement des muscles adducteurs et des abdominaux ainsi qu'exercices de coordination et programme de retour à la course) est plus efficace que les traitements passifs (laser, stimulation électrique nerveuse, massage transverse profond (MTP), étirement des muscles adducteurs).

De même, le traitement multimodal (technique de thérapie manuelle, thermothérapie au niveau des adducteurs, étirement manuel spécifique des muscles adducteurs et programme de retour à la course) raccourcit le temps avant le retour au sport par rapport à la thérapie active simple.

Le succès du traitement est défini par le pourcentage de patients guéris, le pourcentage de satisfaction (bonne ou excellente) du patient, la diminution de la douleur, l'amélioration des scores fonctionnels et le pourcentage de patients de retour au sport. Il est de $84.1 \%$ (SD 16.8, range 27-100\%) dans les groupes d'intervention et de 48.7\% (SD 29.9, range 8-93\%) dans les groupes contrôle. Dans les groupes d'intervention, 90.6\% des patients (SD 11.2, range 49-100\%) sont retournés au sport contre 45.8\% des athlètes (SD 21.9, range 14-64\%) dans les groupes contrôle. Le retour au sport s'est fait en moyenne à 11.3 semaines (SD 8.1, range 1-38 weeks) dans les groupes d'intervention et seulement à 25.6 semaines (SD 5.9, range 2-17 weeks) dans les groupes contrôle.

\section{Conclusion :}

Les résultats de la revue systématique de Serner et al. (2015) démontrent de meilleurs résultats pour la thérapie active que la thérapie passive (Serner et al., 2015). De même, le traitement multimodal est plus efficace que la thérapie active simple. 
Il est également à noter que dans les trois études (Almeida, Machotka et Serner), aucune complication n'a été mise en évidence suite au traitement conservateur (Almeida et al., 2013 ; Machotka et al., 2009 ; Serner et al., 2015).

\section{Discussion}

L'objectif de notre revue «panorama » était de répertorier les différents traitements conservateurs dans le cadre des pubalgies de surcharge chez les athlètes et d'en évaluer l'efficacité.

Plusieurs traitements comme le renforcement musculaire (Almeida et al., 2013), la thérapie active par l'exercice (Almeida et al., 2013; Machotka et al., 2009), la physiothérapie conventionnelle telle que la thermothérapie ou les étirements (Almeida et al., 2013 ; Serner et al., 2015), la thérapie multimodale comprenant une technique de thérapie manuelle et des exercices actifs (Almeida et al., 2013 ; Serner et al., 2015) ou encore un programme de retour à la course (Almeida et al., 2013 ; Machotka et al., 2009 ; Serner et al., 2015), ont démontré leurs effets positifs sur la douleur (Almeida et al., 2013 ; Machotka et al., 2009 ; Serner et al., 2015), la force (Machotka et al., 2009), le retour au sport (Almeida et al., 2013 ; Machotka et al., 2009 ; Serner et al., 2015), le succès du traitement (Almeida et al., 2013 ; Serner et al., 2015), l'évaluation subjective par le patient (Almeida et al., 2013), le pourcentage de patients de retour au sport (Almeida et al., 2013 ; Serner et al., 2015) et le temps jusqu'au retour au sport (Almeida et al., 2013 ; Serner et al., 2015).

Ces résultats nous ont permis d'établir des recommandations thérapeutiques pratiques pour le physiothérapeute.

\subsection{Recommandations GRADE}

Nous pouvons donc faire les recommandations suivantes :

- Le traitement conservateur est efficace pour traiter les pubalgies de surcharge. 
- La thérapie active par l'exercice est supérieure à la thérapie passive. C'est cependant la thérapie multimodale qui démontre les meilleurs résultats et devrait donc être la modalité choisie par le physiothérapeute pour la meilleure efficacité.

\subsection{Force des recommandations}

Nous avons évalué dans le tableau ci-dessous la force des recommandations des trois revues systématiques incluses dans notre travail d'après l'échelle proposée par la Haute Autorité de la santé (Dhénain, 2013).

\begin{tabular}{|c|c|c|c|}
\hline \multirow{3}{*}{$\begin{array}{l}\text { Niveau de preuve } \\
\text { scientifique fourni } \\
\text { par la littérature }\end{array}$} & Almeida (2013) & Machotka (2009) & Serner (2009) \\
\hline & Niveau 1 & Niveau 3 & Niveau 3 \\
\hline & $\begin{array}{l}2 \text { randomised } \\
\text { controlled trial }(\mathrm{RCT})= \\
1\end{array}$ & $\begin{array}{l}1 \mathrm{RCT}=1 \\
2 \text { rapports de cas }=3 \\
2 \text { séries de cas }=4\end{array}$ & $\begin{array}{l}3 \mathrm{RCT}=1 \\
3 \text { séries de cas }=4 \\
3 \text { revues } \\
\text { systématiques (RS) } \\
\text { avec plusieurs types } \\
\text { d'études = } 2\end{array}$ \\
\hline \multirow{2}{*}{$\begin{array}{l}\text { Grade de } \\
\text { recommandations }\end{array}$} & Grade A & Grade B & Grade B \\
\hline & $\begin{array}{l}\text { "Statistiquement } \\
\text { significatif » }\end{array}$ & $\begin{array}{l}\text { «Evidence } \\
\text { modérée » }\end{array}$ & $\begin{array}{l}\text { «Evidence } \\
\text { modérée » }\end{array}$ \\
\hline Thérapies proposées & $\begin{array}{l}\text { Physiothérapie } \\
\text { conventionnelle : } \\
\text { - } \quad \text { Electrothérapie } \\
\text { (TENS et laser) } \\
\text { - MTP } \\
\text { Etirements des } \\
\text { muscles de la } \\
\text { hanche, } \\
\text { adducteurs et } \\
\text { ischio-jambiers) } \\
\text { Thérapie active par } \\
\text { l'exercice : } \\
\text { (3x/semaine à } \\
\text { domicile)6 } \\
\text { semaines } \\
\text { d'exercices en } 2 \\
\text { modules } \\
\text { progressifs } \\
\text { (renforcement } \\
\text { des muscles } \\
\text { adducteurs et } \\
\text { abdominaux, } \\
\text { exercices } \\
\text { d'équilibre et de } \\
\text { coordination) et } \\
\text { du vélo } \\
\text { Puis programme } \\
\text { de retour à la } \\
\text { course en } 3 \\
\text { phases : jogging } \\
\text { lent puis sprint } \\
\text { en ligne droite }\end{array}$ & $\begin{array}{l}\text { Thérapie active par } \\
\text { l'exercice : } \\
\text { - } \quad \text { Repos relatif } \\
\text { - } \quad \text { Etirements et } \\
\text { flexibilité des } \\
\text { adducteurs et } \\
\text { muscles de la } \\
\text { hanche } \\
\text { - } \quad \text { Renforcement } \\
\text { des muscles } \\
\text { adducteurs et } \\
\text { abdominaux } \\
\text { Equilibre et } \\
\text { proprioception } \\
\text { de la ceinture } \\
\text { pelvienne } \\
\text { Coordination } \\
\text { - } \text { Vélo } \\
\text { Programme de } \\
\text { retour à la } \\
\text { course }\end{array}$ & $\begin{array}{l}\text { Divers traitements } \\
\text { conservateurs } \\
\text { (thérapie active et } \\
\text { passive) } \\
\text { - } \quad \text { Renforcement } \\
\text { des muscles } \\
\text { adducteurs et } \\
\text { abdominaux } \\
\text { - } \quad \text { Equilibre } \\
\text { - } \quad \text { Coordination } \\
\text { - Stretching des } \\
\text { adducteurs } \\
\text { - Vélo ou jogging } \\
\text { - lent } \\
\text { Programme de } \\
\text { retour à la } \\
\text { - } \quad \text { Massage } \\
\text { Electrothérapie } \\
\text { (TENS, laser) }\end{array}$ \\
\hline
\end{tabular}




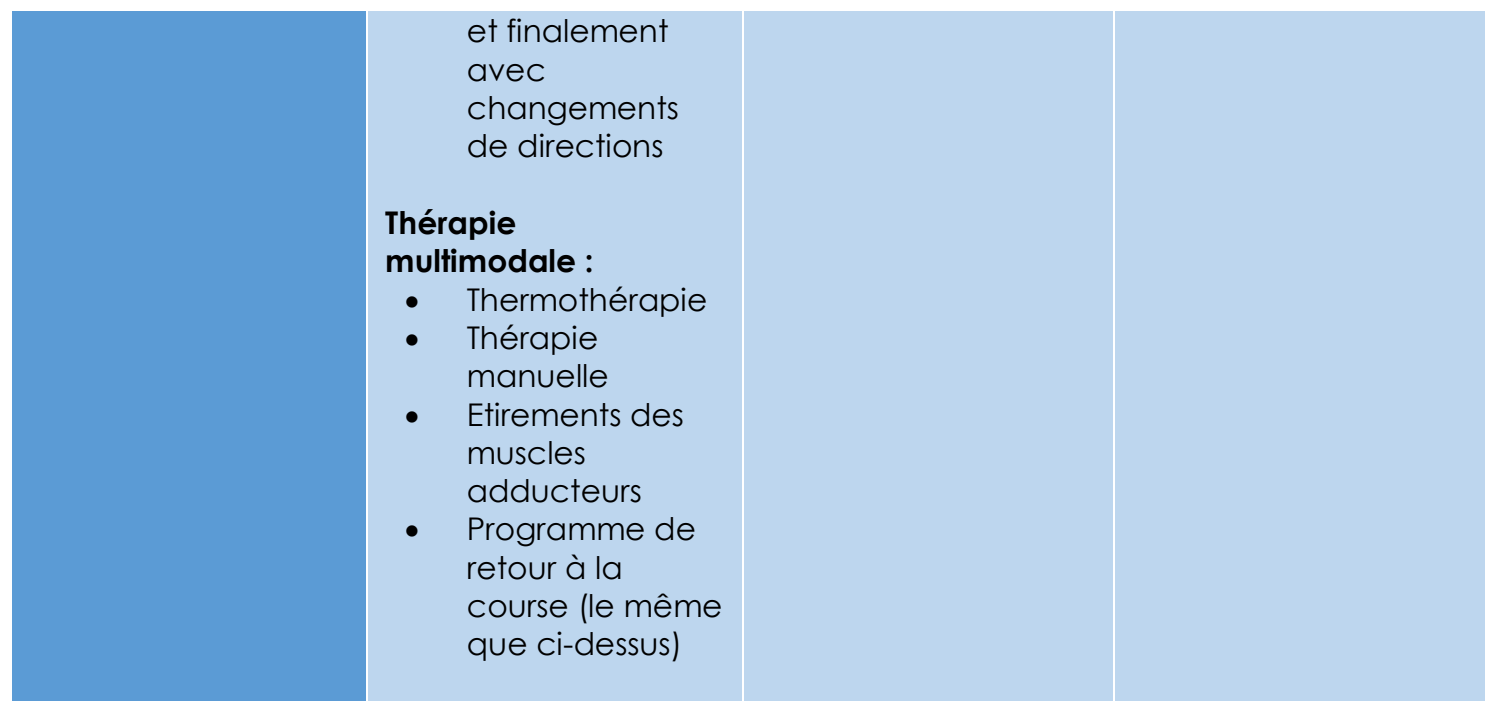

Tableau 4 : Force des recommandations inspiré de l'échelle proposée par la HAS (Barras et Lutz, 2017)

Sur le même modèle que pour les revues systématiques ci-dessus, nous avons évalué la force de nos recommandations. Les trois revues systématiques incluses comprenant également d'autres types d'articles que des études randomisées contrôlées, le niveau de preuve scientifique est de niveau 2. La mise en commun des grades de recommandation des études d'Almeida, Machotka et Serner nous permet d'attribuer le grade $\mathrm{B}$ à notre revue «panorama ». Selon le groupe GRADE, « la force d'une recommandation reflète la confiance que l'on peut avoir dans le fait que les effets souhaitables d'une intervention l'emportent sur les effets indésirables ». Les résultats positifs étant confirmés dans les études incluses et aucune complication due au traitement conservateur n'ayant été observée, notre groupe de travail est confiant dans le fait que les effets positifs l'emportent et nous définissons donc la force de nos recommandations comme forte.

\subsection{Forces et limites de notre étude}

Les forces de notre étude sont l'utilisation des guidelines Prisma, de la check-list AMSTAR ainsi que l'enregistrement dans Prospero. La méthodologie stricte que nous avons appliquée garantit la qualité de notre revue systématique. L'utilisation d'une équation de recherche avec des mots clés et MeshTerms ainsi que la recherche dans plusieurs bases de données et la littérature grise nous ont permis d'identifier un maximum de revues systématiques dans la littérature.

Nous avons aussi identifié certaines limites à notre étude. 
Il faut d'abord se demander si nos recherches ont véritablement permis d'identifier tous les articles pertinents dans la littérature; si ce n'était pas le cas, nous pourrions évoquer un biais de sélection.

La force de nos recommandations est limitée par le manque d'études randomisées contrôlées incluses dans les revues systématiques sélectionnées ainsi que par la qualité méthodologique de ces études, qualifiée de pauvre. Il ne faut cependant pas oublier que la qualité de l'étude ne définit pas forcément la qualité des recommandations qui en sont tirées, comme le rappelle la Haute Autorité de la Santé (HAS) (Dhénain, 2013).

Biais de représentativité : Une autre limitation des résultats est la petite taille d'échantillon qui se retrouve dans la majorité des études sur les traitements conservateurs des pubalgies, ce qui augmente potentiellement le risque de biais de représentativité.

Biais de performance : À l'exception de l'étude de Hölmich et al. (1999), aucune étude n'utilise de protocole préétabli, ce qui rend difficile la comparaison des résultats entre les études mais aussi la réplication des interventions dans la pratique (P. Hölmich et al., 1999). En effet, dans la majorité des études, la prise en charge dépend de l'expérience du physiothérapeute. Machotka (2009) cite comme exemple la progression du traitement ; pour la prise de décision, certains s'appuient sur l'absence de douleur, d'autres sur le contrôle fonctionnel, etc. (Machotka et al., 2009). La difficulté à réunir les résultats des différentes études est aussi causée par la grande diversité des issues traitées, les nombreuses pathologies incluses, les co-interventions, la variation des suivis, le manque de données sur la population et le déroulement des interventions.

Biais de mesure : Il existe un manque de détails (durée de la thérapie, fréquence, intensité, résistance, etc.) concernant le traitement appliqué dans les études, ce qui constitue un biais de mesure.

Biais d'interprétation : Serner (2015) et Almeida (2013) rappellent tous les deux l'importance d'être attentif au risque de biais d'interprétation qui peut découler de l'analyse des résultats et que l'on ne peut totalement exclure pour notre revue «panorama » au vu de la faible qualité des études incluses (Almeida et al., 2013 ; Serner et al., 2015).

Le manque de données numériques et de résultats objectivables dans les revues systématiques sur le traitement conservateur des pubalgies empêche la réalisation d'une méta-analyse. 


\subsection{Pistes pour de futures études}

Il est nécessaire de réaliser de nouvelles études randomisées contrôlées afin de combler le manque de littérature et d'améliorer la qualité des données à analyser.

Dans les études futures, il est important de donner plus de détails sur le déroulement du traitement, tels que le nombre de répétitions, l'intensité, la fréquence, les exercices pratiqués, etc. Le mieux serait d'utiliser un protocole préétabli comme dans l'étude d'Hölmich (P. Hölmich et al., 1999).

Il serait également intéressant d'établir des sous-groupes selon les pathologies et/ou les degrés d'atteinte, ce qui permettrait une analyse sensitive plus précise et potentiellement des recommandations adaptées à chaque patient.

Machotka (2009) suggère que la supervision par un physiothérapeute et les cointerventions pourraient avoir une influence sur les résultats d'un traitement, il faudrait donc également y prêter attention lors de futures études (Machotka et al., 2009).

\section{Conclusion}

Cette revue «panorama » qualitative analyse les traitements conservateurs des pubalgies afin d'en extraire des recommandations. Les recherches réalisées ont permis d'identifier trois revues systématiques répondant à notre question de recherche. À partir de ces quatre articles, nous avons pu émettre des recommandations thérapeutiques pratiques pour le physiothérapeute au quotidien : préférer la thérapie multimodale aux autres prises en charge conservatives pour les athlètes souffrant de pubalgies.

Nous restons lucides sur le fait que des revues systématiques incluant des études randomisées contrôlées de bonne qualité sont nécessaires pour confirmer la validité de ces recommandations dans le futur. Il est également important de garder un regard critique sur les recommandations afin d'adapter la prise en charge à chaque patient pour de la rendre optimale.

Cette revue systématique de revues systématiques est utile aux physiothérapeutes au quotidien, car il n'existe encore aucune recommandation consensuelle pour la prise en charge de ces patients. Elle amène une vision actuelle sur la prise en charge des pubalgies de surcharge chez l'athlète et démontre que le traitement conservateur a son rôle dans la prise en charge de cette pathologie. 


\section{Références}

Albers, I. S., Zwerver, J., Diercks, R. L., Dekker, J. H., \& Van den Akker-Scheek, I. (2016). Incidence and prevalence of lower extremity tendinopathy in a Dutch general practice population: a cross sectional study. BMC Musculoskeletal Disorders, 17. https://doi.org/10.1186/s12891-016-0885-2

Almeida, M. O., Silva, B. N. G., Andriolo, R. B., Atallah, A. N., \& Peccin, M. S. (2013) Conservative interventions for treating exercise-related musculotendinous, ligamentous and osseous groin pain. The Cochrane database of systematic reviews, (6), CD009565. https://doi.org/10.1002/14651858.CD009565.pub2

Bisciotti, G. N., Auci, A., Di Marzo, F., Galli, R., Pulici, L., Carimati, G., ... Volpi, P. (2015). Groin pain syndrome: an association of different pathologies and a case presentation. Muscles, ligaments and tendons journal, 5(3), 214-222. https://doi.org/10.11138/mltj/2015.5.3.214

Bouvard, M., Dorochenko, P., Lanusse, P., \& Duraffour, H. (2004, septembre). La pubalgie du sportif - stratégie thérapeutique. EM-Consulte. Repéré à http://www.emconsulte.com/article/86663/la-pubalgie-du-sportif-?-strategie-therapeutique

Caudill, P., Nyland, J., Smith, C., Yerasimides, J., \& Lach, J. (2008). Sports hernias: a systematic literature review. British journal of sports medicine, 42(12), 954-964. https://doi.org/10.1136/bjsm.2008.047373

Cowan, S. M., Schache, A. G., Brukner, P., Bennell, K. L., Hodges, P. W., Coburn, P., \& Crossley, K. M. (2004). Delayed onset of transversus abdominus in long-standing groin pain. Medicine and science in sports and exercise, 36(12), 2040-2045.

de Larra y Arnaiz, I. M. (2011, 16 octobre). Ostéopathie dynamique du pubis. Traitement de kinésithérapie. - Artículo de Fisioterapia. eFisioterapie.net. Repéré à http://www.efisioterapia.net/fr/articles/osteopathie-dynamique-du-pubis-traitementkinesitherapie

de Sa, D., Holmich, P., Phillips, M., Heaven, S., Simunovic, N., Philippon, M. J., \& Ayeni, O. R. (2016). Athletic groin pain: a systematic review of surgical diagnoses, investigations and treatment. British journal of sports medicine. https://doi.org/10.1136/bjsports-2015095137

Dhénain, M. (2013, avril). Haute Autorité de Santé - Niveau de preuve et gradation des recommandations de bonne pratique - État des lieux. Repéré à http://www.hassante.fr/portail/jcms/c_1600564/fr/niveau-de-preuve-et-gradation-desrecommandations-de-bonne-pratique-etat-des-lieux

Drew, M. K. (2015). Some athletes are immature...skeletally. British journal of sports medicine, 49(12), 766. https://doi.org/10.1136/bjsports-2015-094660

Ekstrand, J., \& Hilding, J. (1999). The incidence and differential diagnosis of acute groin injuries in male soccer players. Scandinavian Journal of Medicine \& Science in Sports, 9(2), 98-103. https://doi.org/10.1111/j.1600-0838.1999.tb00216.x

Elattar, O., Choi, H.-R., Dills, V. D., \& Busconi, B. (2016). Groin Injuries (Athletic Pubalgia) and Return to Play. Sports Health: A Multidisciplinary Approach, 8(4), 313-323. https://doi.org/10.1177/1941738116653711

Ellsworth, A. A., Zoland, M. P., \& Tyler, T. F. (2014). Athletic pubalgia and associated rehabilitation. International journal of sports physical therapy, 9(6), 774.

Falvey, E. C., Franklyn-Miller, A., \& McCrory, P. R. (2009). The groin triangle: a pathoanatomical approach to the diagnosis of chronic groin pain in athletes. British journal of sports medicine, 43(3), 213-220. https://doi.org/10.1136/bjsm.2007.042259

Farber, A. J., \& Wilckens, J. H. (2007). Sports hernia: diagnosis and therapeutic approach. The Journal of the American Academy of Orthopaedic Surgeons, 15(8), 507-514.

Grairi, K. (2011). La pubalgie du sportif- Approche thérapeutique et préventive du footballeur professionnel. Revue de la littérature. Haute École Louvain en Hainaut, Louvain en Hainaut. Repéré à http://www.irbms.com/download/documents/karima-grairi-pubalgiesfootballeur.pdf

Guyatt, G., Santesso, N., \& Akl, E. (2004). GRADE handbook for grading the quality of evidence and the strength of recommendations version 3.2. Holger Schünemann, Jan Brozek, Andrew Oxman. Repéré à http://www.who.int/hiv/topics/mtct/grade_handbook.pdf

Hackney, R. G. (1993). The sports hernia: a cause of chronic groin pain. British Journal of Sports Medicine, 27(1), 58-62. https://doi.org/10.1136/bjsm.27.1.58 
Holmich, P., Larsen, K., Krogsgaard, K., \& Gluud, C. (2010). Exercise program for prevention of groin pain in football players: a cluster-randomized trial. Scandinavian journal of medicine \& science in sports, 20(6), 814-821. https://doi.org/10.1111/j.16000838.2009.00998.x

Hölmich, P., Uhrskou, P., Ulnits, L., Kanstrup, I. L., Nielsen, M. B., Bjerg, A. M., \& Krogsgaard, K. (1999). Effectiveness of active physical training as treatment for long-standing adductor-related groin pain in athletes: randomised trial. Lancet (London, England), 353(9151), 439-443. https://doi.org/10.1016/S0140-6736(98)03340-6

Hölmich, Per, Nyvold, P., \& Larsen, K. (2011). Continued Significant Effect of Physical Training as Treatment for Overuse Injury: 8- to 12-Year Outcome of a Randomized Clinical Trial. The American Journal of Sports Medicine, 39(11), 2447-2451. https://doi.org/10.1177/0363546511416075

Hölmich, Per, Uhrskou, P., Ulnits, L., Kanstrup, I.-L., Nielsen, M. B., Bjerg, A. M., \& Krogsgaard, K. (1999). Effectiveness of active physical training as treatment for long-standing adductor-related groin pain in athletes: randomised trial. The Lancet, 353(9151), 439-443. https://doi.org/10.1016/S0140-6736(98)03340-6

Ishøi, L., Sørensen, C. N., Kaae, N. M., Jørgensen, L. B., Hölmich, P., \& Serner, A. (2016). Large eccentric strength increase using the Copenhagen Adduction exercise in football: A randomized controlled trial. Scandinavian Journal of Medicine \& Science in Sports, 26(11), 1334-1342. https://doi.org/10.1111/sms.12585

Joesting, D. R. (2002). Diagnosis and treatment of sportsman's hernia. Current Sports Medicine Reports, 1(2), 121-124.

Kachingwe, A. F., \& Grech, S. (2008). Proposed algorithm for the management of athletes with athletic pubalgia (sports hernia): a case series. The Journal of orthopaedic and sports physical therapy, 38(12), 768-781. https://doi.org/10.2519/jospt.2008.2846

Khan, K., \& Al Tunaiji, H. (2016). Chronic groin pain among athletes: diagnostic approach. Aspetar Sports Medicine Journal. Repéré à http://www.aspetar.com/journal/viewarticle.aspx?id=3\#.WCl4fPnhBPY

Kleist, P. (2009). Le principe de l'intention-to-treat. Forum Med Suisse. Repéré à http://medicalforum.ch/docs/smf/archiv/fr/2009/2009-25/2009-25-011.pdf

Langhout, R., Tak, I., \& Glasgow, P. (2016). Leistenprobleme Ein unfassender Ansatz aus sportphysiotherapeutischer Sicht. Mai 2016, (2-16), p.64-71. https://doi.org/10.1055/s0042-104337

Larson, C. M., \& Lohnes, J. H. (2002). Surgical management of athleticpubalgia. Operative Techniques in Sports Medicine, 10(4), 228-232. https://doi.org/10.1053/otsm.2002.35874

Larson, D. W., \& Farley, D. R. (2002). Spigelian Hernias: Repair and Outcome for 81 Patients. World Journal of Surgery, 26(10), 1277-1281. https://doi.org/10.1007/s00268-002-66050

Leadbetter, W. B. (1992). Cell-matrix response in tendon injury. Clinics in Sports Medicine, 11(3), 533-578.

LeBlanc, K. E., \& LeBlanc, K. A. (2003). Groin pain in athletes. Hernia, 7(2), 68-71. https://doi.org/10.1007/s10029-002-0105-x

Leitner, D. W. M. D., \& Sherwood, R. C. M. D. (1983). Inguinal Lymphocele as a Complication of Thighplasty. : Plastic and Reconstructive Surgery. Repéré à http://journals.Iww.com/plasreconsurg/Fulltext/1983/12000/Inguinal_Lymphocele_as_a_ Complication_of.32.aspx

Machotka, Z., Kumar, S., \& Perraton, L. G. (2009). A systematic review of the literature on the effectiveness of exercise therapy for groin pain in athletes. BMC Sports Science, Medicine and Rehabilitation, 1, 5. https://doi.org/10.1186/1758-2555-1-5

McAleer, S. S., Gille, J., Bark, S., \& Riepenhof, H. (2015). Management of chronic recurrent osteitis pubis/pubic bone stress in a Premier League footballer: Evaluating the evidence base and application of a nine-point management strategy. Physical therapy in sport: official journal of the Association of Chartered Physiotherapists in Sports Medicine, 16(3), 285-299. https://doi.org/10.1016/j.ptsp.2015.04.003

McCarthy, A., \& Vicenzino, B. (2003). Treatment of osteitis pubis via the pelvic muscles. Manual Therapy, 8(4), 257-260. https://doi.org/10.1016/S1356-689X(03)00054-7

Meyers, W. C., McKechnie, A., Philippon, M. J., Horner, M. A., Zoga, A. C., \& Devon, O. N. (2008). Experience with « sports hernia » spanning two decades. Annals of Surgery, 248(4), 656-665. https://doi.org/10.1097/SLA.0b013e318187a770 
Micheletti, L., Preti, M., Zola, P., Valentino, M. C. Z., Bocci, C., \& Bogliatto, F. (1998). A proposed glossary of terminology related to the surgical treatment of vulvar carcinoma. Cancer, 83(7), 1369-1375. https://doi.org/10.1002/(SICI)10970142(19981001)83:7<1369::AID-CNCR14>3.0.CO;2-2

Moher, D., Liberati, A., Tetzlaff, J., \& Altman, D. G. (2009). Preferred Reporting Items for Systematic Reviews and Meta-Analyses: The PRISMA Statement. PLoS Medicine, 6(7). https://doi.org/10.1371/journal.pmed.1000097

Morelli, V., \& Smith, V. (2001). Groin injuries in athletes. American Family Physician, 64(8), 1405-1414.

Munegato, D., Bigoni, M., Gridavilla, G., Olmi, S., Cesana, G., \& Zatti, G. (2015). Sports hernia and femoroacetabular impingement in athletes: A systematic review. World journal of clinical cases, 3(9), 823-830. https://doi.org/10.12998/wjcc.v3.i9.823

Paajanen, H., Ristolainen, L., Turunen, H., \& Kujala, U. M. (2010). Prevalence and etiological factors of sport-related groin injuries in top-level soccer compared to non-contact sports. Archives of Orthopaedic and Trauma Surgery, 131(2), 261-266. https://doi.org/10.1007/s00402-010-1169-1

Pizzari, T., Coburn, P. T., \& Crow, J. F. (2008). Prevention and management of osteitis pubis in the Australian Football League: A qualitative analysis. Physical Therapy in Sport, 9(3), 117-125. https://doi.org/10.1016/j.ptsp.2008.06.002

Pruvost, J. (2015, 27 mai). Le site au coeur de la médecine du sport. La médecine du sport. Repéré à http://www.lamedecinedusport.com/traumatologie/premiere-journeeeuropeenne-sur-la-pubalgie-les-points-forts-du-congres/

Puig, P. L., Trouve, P., \& Savalli, L. (2004). [Pubalgia: from diagnosis to return to the sports field]. Annales de readaptation et de medecine physique : revue scientifique de la Societe francaise de reeducation fonctionnelle de readaptation et de medecine physique, 47(6), 356-364. https://doi.org/10.1016/j.annrmp.2004.05.003

Quinn, A. (2010). Hip and Groin Pain: Physiotherapy and Rehabilitation Issues. The Open Sports Medicine Journal, 4, 93-107. 2010, 4(93-107).

Radojević, S. (1962). ANATOMIE CHIRURGICALE DE LA RÉGION INGUINALE. Cells Tissues Organs, 50(3), 208-263. https://doi.org/10.1159/000141904

Rodriguez, C., Miguel, A., \& Lima, H. (2001). Osteitis pubis syndrome in the professional soccer athlete: A case report - ProQuest. Repéré à http://search.proquest.com/openview/01702e226304e0ac2b1b672af4ed408f/1?pqorigsite $=$ gscholar $\& \mathrm{cbl}=47878$

Sedaghati, P., Alizadeh, M.-H., Shirzad, E., \& Ardjmand, A. (2013). Review of sport-induced groin injuries. Trauma monthly, 18(3), 107.

Serner, A., van Eijck, C. H., Beumer, B. R., Holmich, P., Weir, A., \& de Vos, R.-J. (2015). Study quality on groin injury management remains low: a systematic review on treatment of groin pain in athletes. British journal of sports medicine, 49(12), 813. https://doi.org/10.1136/bjsports-2014-094256

Shea, B. J., Grimshaw, J. M., Wells, G. A., Boers, M., Andersson, N., Hamel, C., ... Bouter, L. M. (2007). Development of AMSTAR: a measurement tool to assess the methodological quality of systematic reviews. BMC Medical Research Methodology, 7(1). https://doi.org/10.1186/1471-2288-7-10

Smith, V., Devane, D., Begley, C. M., \& Clarke, M. (2011). Methodology in conducting a systematic review of systematic reviews of healthcare interventions. BMC Medical Research Methodology, 11, 15. https://doi.org/10.1186/1471-2288-11-15

St-Onge, E., MacIntyre, I. G., \& Galea, A. M. (2015). Multidisciplinary approach to non-surgical management of inguinal disruption in a professional hockey player treated with plateletrich plasma, manual therapy and exercise: a case report. The Journal of the Canadian Chiropractic Association, 59(4), 390-397.

Sugimoto, D., Mattacola, C. G., Mullineaux, D. R., Palmer, T. G., \& Hewett, T. E. (2014). Comparison of Isokinetic Hip Abduction and Adduction Peak Torques and Ratio Between Sexes. Clinical journal of sport medicine : official journal of the Canadian Academy of Sport Medicine, 24(5), 422-428. https://doi.org/10.1097/JSM.0000000000000059

Trofa, D. P., Mayeux, S. E., Parisien, R. L., Ahmad, C. S., \& Lynch, T. S. (2017). Mastering the Physical Examination of the Athlete's Hip. American Journal of Orthopedics (Belle Mead, N.J.), 46(1), 10-16.

Verrall, G. M., Slavotinek, J. P., Fon, G. T., \& Barnes, P. G. (2007). Outcome of Conservative Management of Athletic Chronic Groin Injury Diagnosed as Pubic Bone Stress Injury. 
The American Journal of Sports Medicine, 35(3), 467-474. https://doi.org/10.1177/0363546506295180

Waldén, M., Hägglund, M., \& Ekstrand, J. (2015). The epidemiology of groin injury in senior football: a systematic review of prospective studies. British Journal of Sports Medicine, 49(12), 792-797. https://doi.org/10.1136/bjsports-2015-094705

Weir, A., Jansen, J. A. C. G., van de Port, I. G. L., Van de Sande, H. B. A., Tol, J. L., \& Backx, F. J. G. (2011). Manual or exercise therapy for long-standing adductor-related groin pain: A randomised controlled clinical trial. Manual Therapy, 16(2), 148-154. https://doi.org/10.1016/j.math.2010.09.001

Weir, Adam, Brukner, P., Delahunt, E., Ekstrand, J., Griffin, D., Khan, K. M., ... Holmich, P. (2015). Doha agreement meeting on terminology and definitions in groin pain in athletes. British journal of sports medicine, 49(12), 768-774. https://doi.org/10.1136/bjsports-2015-094869

Wollin, M., \& Lovell, G. (2006). Osteitis pubis in four young football players: A case series demonstrating successful rehabilitation. Physical Therapy in Sport, 7(3), 153-160. https://doi.org/10.1016/j.ptsp.2006.03.005

Yuill, E. A., Pajaczkowski, J. A., \& Howitt, S. D. (2012). Conservative care of sports hernias within soccer players: a case series. Journal of bodywork and movement therapies, 16(4), 540-548. https://doi.org/10.1016/j.jbmt.2012.04.004

Zaugg, V., Savoldelli, V., Sabatier, B., \& Durieux, P. (2014). Améliorer les pratiques et l'organisation des soins: méthodologie des revues systématiques. 2014, 26(5), 160. 


\section{Liste des illustrations}

Figure 1 : Illustration de Cornford 3

Figure 2 : Flow chart de la sélection des articles 12

Figure 3 : Evaluation de la qualité selon AMSTAR 13

Tableau 1 : Etiologies des douleurs chroniques à l'aine chez les athlètes 4

Tableau 2 : Stades de tendinopathies 5

Tableau 3 : Description des études incluses 14

Tableau 4 : Force des recommandations 20-21 


\section{Annexes}

\section{Annexe I : Diagnostics différentiels}

\begin{tabular}{|l|}
\hline Orthopaedic causes \\
\hline Bone/joint \\
Femoroacetabular impingement (FAl) \\
Acetabular labral tears \\
Osteitis pubis \\
Stress fractures: Pubic ramus/femoral neck \\
Degenerative hip joint disease \\
Avascular necrosis of the femoral head \\
Slipped capital femoral epiphysis \\
Avulsion fracture: Anterior superior iliac spine/ \\
anterior inferion spine/ischium \\
\hline Muscle \\
Athletic pubalgia \\
Hockey player's syndrome \\
Rectus femoris strain/tear \\
Adductor strain/tendonitis \\
lliopsoas strain/tear \\
Rectus abdominis strain/tear: Muscle contusion \\
Gracilis syndrome \\
Snapping hip syndrome \\
\hline Neurologic \\
Nerve entrapment: llioinguinal/obturator neuropathy \\
Lumbar radiculopathy \\
\hline Other orthopaedic \\
Bursitis \\
Bone/soft tissue neoplasm of hip/pelvis neoplasms : \\
Bone/soft tissue \\
Herniated nucleus pulposus \\
Seronegative spondyloarthropathy \\
\hline Non orthopaedic causes \\
\hline Hernia: Inguinal/femoral \\
\hline Urologic \\
Prostatitis/epididymitis/urethritis/urinary tract \\
infection \\
Testicular neoplasm \\
Ureteral colic \\
Hydrocele/varicocele \\
\hline Gynecologic \\
Endometriosis \\
Pelvic inflammatory disease \\
Ovarian cyst \\
\hline Gastrointestinal \\
Rectal/colon neoplasm \\
Inflammatory bowel disease \\
Diverticulitis \\
\hline Tateas:Dagnstics dffentls \\
\hline
\end{tabular}

Tableau 5 : Diagnostics différentiels de la pubalgie (Elattar et al., 2016) 


\section{Annexe II : Extraction des données (Almeida)}

\begin{tabular}{|c|c|}
\hline Nom de l'étude & $\begin{array}{l}\text { Conservative interventions for treating exercise- } \\
\text { related musculotendinous, ligamentous and } \\
\text { osseous groin pain (Review) }\end{array}$ \\
\hline Date de publication & 2013 \\
\hline Nom des auteurs & $\begin{array}{l}\text { Almeida MO, Silva BNG, Andriolo RB, Atallah ÁN, } \\
\text { Peccin MS }\end{array}$ \\
\hline Design & Revue systématique \\
\hline $\begin{array}{l}\text { Qualité } \\
\text { (évalué avec AMSTAR) }\end{array}$ & $11 / 11$ \\
\hline Etudes incluses & $\begin{array}{l}\text { - Continued significant effect of physical training as } \\
\text { treatment for overuse injury: } 8 \text { - to 12-year outcome } \\
\text { of a randomized clinical trial } \\
\text { (Hölmich et al. 1999) } \\
\text { RCT } \\
\text { - Manual or exercise therapy for longstanding } \\
\text { adductor-related groin pain: a randomised } \\
\text { controlled clinical trial (Weir et al. 2011) } \\
\text { RCT }\end{array}$ \\
\hline Population & $\begin{array}{l}\text { Hölmich } 1999 \\
\text { Athlètes atteints de pubalgie depuis au moins } 2 \\
\text { mois désirant continuer le sport au même niveau } \\
\text { de compétition qu'avant la blessure. } \\
\text { Âge : } 18 \text { à } 50 \text { ans } \\
\text { Genre : hommes } \\
\text { Weir } 2011 \\
\text { Athlètes souffrant de douleurs au niveau de l'aine } \\
\text { après le sport depuis au moins } 2 \text { mois désirant } \\
\text { continuer le sport au même niveau qu'avant la } \\
\text { blessure. } \\
\text { Âge : } 18 \text { à } 50 \text { ans } \\
\text { Genre: } 53 \text { hommes et } 1 \text { femme }\end{array}$ \\
\hline Pathologie(s) & $\begin{array}{l}\text { Douleurs à l'aine liées aux adducteurs depuis au } \\
\text { moins } 2 \text { mois }\end{array}$ \\
\hline Intervention & $\begin{array}{l}\text { Toutes formes de traitement conservateur tels } \\
\text { que: } \\
\text { - le renforcement des muscles stabilisateurs de la } \\
\text { hanche et du pelvis } \\
\text { - stretching des muscles de la hanche } \\
\text { - électrothérapie (laser, ultrasons) } \\
\text { - thérapie manuelle } \\
\text { Hölmich } \\
\text { Thérapie active par l'exercice (renforcement des } \\
\text { adducteurs et abdominaux, exercices de } \\
\text { coordination) }\end{array}$ \\
\hline
\end{tabular}




\begin{tabular}{|c|c|}
\hline & $\begin{array}{l}\text { Physiothérapie conventionnelle consistant en } \\
\text { modalités passives (massage transverse profons, } \\
\text { laser, TENS et étirement des adducteurs, ischio- } \\
\text { jambiers et des muscles de la hanche). } \\
\text { Weir } \\
\text { Traitement multimodal (thermothérapie, étirement } \\
\text { des adducteurs) } \\
\text { Traitement actif par l'exercice (renforcement des } \\
\text { adducteurs et abdominaux, exercices de } \\
\text { coordination) }\end{array}$ \\
\hline Nombre de sujets & 122 \\
\hline Groupe contrôle & $\begin{array}{l}\text { Hölmich : physiothérapie conventionnelle } \\
\text { Weir : Thérapie active par l'exercice }\end{array}$ \\
\hline Issues & $\begin{array}{l}\text { Primaires } \\
\text { - Diminution de la douleur au repos, à la palpation } \\
\text { et durant l'activité physique (VAS, échelle } \\
\text { numérique, ...) } \\
\text { - Fonction (Short Form-36 (SF-36) } \\
\text { - Succès du traitement } \\
\text { Secondaires } \\
\text { - Evaluation subjective globale par le patient } \\
\text { (beaucoup mieux, mieux, pas mieux, pire et } \\
\text { vraiment pire) } \\
\text { - Retour au sport ou aux activités normales } \\
\text { - Evénements indésirables (ex: brûlure par la } \\
\text { cryothérapie) } \\
\text { - Temps jusqu'au retour au sport }\end{array}$ \\
\hline Suivi & $\begin{array}{l}\text { Hölmich Evaluation à } 16 \text { semaines et à long terme } \\
\text { (8-12 ans) } \\
\text { Weir Evaluation à } 16 \text { semaines }\end{array}$ \\
\hline $\begin{array}{l}\text { Une population } \\
\text { importante a-t-elle été } \\
\text { exclue? }\end{array}$ & Les femmes \\
\hline $\begin{array}{l}\text { Manques identifiés/ce } \\
\text { qu'il reste à faire }\end{array}$ & $\begin{array}{l}\text { Besoin d'autres études randomisées contrôlées } \\
\text { pour confirmer/ réfuter les possibles effets } \\
\text { favorables de la thérapie active par l'exercice/ la } \\
\text { thérapie multimodale/ autres traitements } \\
\text { conservateurs. } \\
\text { Besoin d'autres études avec un suivi à long terme } \\
\text { et évaluant la douleur à l'aide d'instruments de } \\
\text { mesure standardisés. } \\
\text { Besoin d'évaluer également la fonction pour éviter } \\
\text { un retour au sport dans de mauvaises conditions. } \\
\text { Pour cela, le Copenhagen Hip and Groin } \\
\text { Outcome Score (Thorborg } 2011 \text { ) qui évalue les } \\
\text { symptômes et la fonction durant les activités de la }\end{array}$ \\
\hline
\end{tabular}


vie quotidienne et du sport peut être une piste d'outil.

Tableau 6 : Extraction des données de Almeida et al. (2013)

\section{Annexe III : Extraction des données (Machotka)}

\begin{tabular}{|c|c|}
\hline Nom de l'étude & $\begin{array}{l}\text { A systematic review of the literature on the } \\
\text { effectiveness of exercise therapy for groin pain in } \\
\text { athletes }\end{array}$ \\
\hline Date de publication & 2009 \\
\hline Nom des auteurs & $\begin{array}{l}\text { Zuzana Machotka, Saravana Kumar and Luke G } \\
\text { Perraton }\end{array}$ \\
\hline Design & Revue systématique de la littérature \\
\hline $\begin{array}{l}\text { Qualité } \\
\text { (évalué avec AMSTAR) }\end{array}$ & $8 / 11$ \\
\hline $\begin{array}{l}\text { Etudes incluses } \\
\text { (titre, auteurs, date, design) }\end{array}$ & $\begin{array}{l}\text { - Effectiveness of active physical training as } \\
\text { treatment for long-standing adductorrelated groin } \\
\text { pain in athletes: randomised trial. } \\
\text { (Holmich P et al. 2009) } \\
\text { RCT } \\
\text { - Treatment of osteitis pubis via the pelvic muscles. } \\
\text { (McCarthy A et Vincenzino B, 2003) } \\
\text { Case report } \\
\text { - Osteitis pubis syndrome in the professional } \\
\text { soccer athlete: a case report. } \\
\text { (Rodriguez C, Miguel A, Lima H, et al. 2001) } \\
\text { Case report } \\
\text { - Osteitis pubis in four young football players: a } \\
\text { case series demonstrating } \\
\text { rehabilitation } \\
\text { (Wollin M et Lovell G, 2006) } \\
\text { Case series } \\
\text { - Outcome of conservative management of } \\
\text { athletic chronic groin injury diagnosed as pubic } \\
\text { stress injury. } \\
\text { (Verrall GM, Slavotinek JP, Fon GT, et al. 2007) } \\
\text { Case series }\end{array}$ \\
\hline Population & $\begin{array}{l}\text { Athlètes se plaignant de douleurs à l'aine } \\
135 \text { hommes } \\
\text { Sports identifiés dans les études: }\end{array}$ \\
\hline
\end{tabular}




\begin{tabular}{|c|c|}
\hline & $\begin{array}{l}\text { Soccer/ gaelic football/ australian rules football \& } \\
\text { running, tennis, European handball, badminton, } \\
\text { ice hockey, basketball, horseriding, rugby }\end{array}$ \\
\hline Pathologie(s) & $\begin{array}{l}\text { Pubalgie = } \\
\text { 1) subjectif : douleur dans la zone de l'iliopsoas et du groupe } \\
\text { des adducteurs ou de la musculature abdominale basse } \\
\text { 2) objectif : douleur à la palpation des adducteurs, de la } \\
\text { musculature abdominale basse, de l'os du pubis ou de la } \\
\text { symphyse pubienne } \\
\text { 3) Tests de longueur ou de force musculaire des adducteurs } \\
\text { positifs } \\
\text { 4) Douleurs ci-dessus affectant une fonction ou une } \\
\text { composante de l'activité de l'athlète } \\
\text { sANs: hernia, piriformis syndrome, sacroiliac } \\
\text { dysfunction, nerve entrapments, referral from the } \\
\text { lumbar spine, rectal or testicular referral and co- } \\
\text { existing fractures of the pelvis or lower limb \& groin } \\
\text { pain associated with any abnormality or } \\
\text { pathology relating to the hip }\end{array}$ \\
\hline Intervention & $\begin{array}{l}\text { Thérapie active par l'exercice } \\
4 / 5 \text { incluent du renforcement through-range } \\
\text { (isocinétique) (F. hanche/ ABD/ ADD/ musculature } \\
\text { abdominale) puis progression vers through-range en } \\
\text { mouvement utilisant des positions fonctionnelles } \\
\text { similaires à celles requises dans leur sport } \\
1 / 5 \text { protocole prédéterminé d'exercices } \\
\text { (4/5 indication for progression = absence of pain during } \\
\text { exercise/ acquisition of functional control/ ability to } \\
\text { complete a functional exercise or a set number of repetitions } \\
\text { of an exercise) }\end{array}$ \\
\hline Nombre de sujets & $\begin{array}{l}\text { Total : } 135 \\
\text { (Hölmich : } 68, \text { McCarthy \& Vicenzino : 1, Rodriguez : } 35 \text { (over } \\
8 \text { years), Wollin \& Lowell }: 4 \text {, Verrall : 27) }\end{array}$ \\
\hline Co-interventions & $\begin{array}{l}\text { - massage } \\
\text { - manipulation => passif }(2 / 5) \\
\text { - médicaments anti-inflammatoires }(1 / 5) \\
\text { - jogging/running }(4 / 5) \\
\text { - vélo }(4 / 5)\end{array}$ \\
\hline Issues & $\begin{array}{l}\text { - Retour au sport } \\
\text { - Tests de longueur musculaire des adducteurs } \\
\text { - Tests de force musculaire des adducteurs } \\
\text { - Douleur }\end{array}$ \\
\hline $\begin{array}{l}\text { Une population } \\
\text { importante a-t-elle été } \\
\text { exclue? }\end{array}$ & Les femmes \\
\hline $\begin{array}{l}\text { Manques identifiés/ ce } \\
\text { qu'il reste à faire }\end{array}$ & $\begin{array}{l}\text { Besoin de revues systématiques avec des groupes } \\
\text { clairement définis selon les diagnostiques et des } \\
\text { détails sur les caractéristiques des exercices. } \\
\text { Aucune étude ne rapportait de manière } \\
\text { appropriée l'intensité de l'intervention. Les } \\
\text { données telles que la résistance, le poids ou la }\end{array}$ \\
\hline
\end{tabular}




\begin{tabular}{|l|l|}
\hline & $\begin{array}{l}\text { perception de l'effort n'étaient pas reportées. } \\
\text { Seules deux études donnent la fréquence des } \\
\text { sessions d'exercice par semaine. }\end{array}$ \\
\hline & \\
\hline
\end{tabular}

Tableau 7 : Extraction des données de Machotka et al. (2009)

Annexe IV : Extraction des données (Serner)

\begin{tabular}{|c|c|}
\hline Nom de l'étude & $\begin{array}{l}\text { Study quality on groin injury management } \\
\text { remains low : a systematic review on treatment } \\
\text { of groin pain in athletes }\end{array}$ \\
\hline Date de publication & 2015 \\
\hline Nom des auteurs & $\begin{array}{l}\text { Andreas Serner, Casper H van Eijck, Berend R } \\
\text { Beumer, Per Hölmich, Adam Weir, Robert-Jan de } \\
\text { Vos }\end{array}$ \\
\hline Design & Revue systématique \\
\hline $\begin{array}{l}\text { Qualité } \\
\text { (évalué avec AMSTAR) }\end{array}$ & $9 / 11$ \\
\hline $\begin{array}{l}\text { Etudes incluses } \\
\text { => pris que les études sur le TTT } \\
\text { conservateur (physio mais pas } \\
\text { injections/ shorts de } \\
\text { compression/ ...) }\end{array}$ & $\begin{array}{l}\text { - Effectiveness of active physical training as } \\
\text { treatment for long-standing adductor-related } \\
\text { groin pain in athletes: randomised trial. } \\
\text { (Holmich P et al. 1999) } \\
\text { - Manual or exercise therapy for long-standing } \\
\text { adductor-related groin pain : a randomised } \\
\text { controlled clinical trial (Weir et al. } 2011 \text { ) } \\
\text { - Outcome of conservative management of } \\
\text { athletic chronic groin injury diagnosed as pubic } \\
\text { bone stress injury } \\
\text { (Verrall et al. 2007) } \\
\text { - Success of nonoperative management of } \\
\text { adductor longus tendon ruptures in National } \\
\text { Football League athletes (Schlegel et al. 2009) } \\
\text { - A manual therapy technique for chronic } \\
\text { adductor-related groin pain in athletes: a case } \\
\text { series } \\
\text { (Weir et al. 2009) } \\
\text { - Treatment of longstanding groin pain in } \\
\text { athletes: a systematic review (Jansen et al. } \\
2008 \text { ) }\end{array}$ \\
\hline
\end{tabular}




\begin{tabular}{|c|c|}
\hline & $\begin{array}{l}\text { - A systematic review of the literature on the } \\
\text { effectiveness of exercise therapy for groin pain } \\
\text { in athletes (Machotka et al. 2009) } \\
\text { - Conservative interventions for treating } \\
\text { exercise-related musculotendinous, } \\
\text { ligamentous and osseus groin pain (Almeida et } \\
\text { al. 2013) } \\
\text { - Short and mid-term results of a comprehensive } \\
\text { treatment program for lonstanding adductor- } \\
\text { related groin pain in athleetes: a case series } \\
\text { (Weir et al. 2010) }\end{array}$ \\
\hline Population & $\begin{array}{l}\text { Athlètes avec un diagnostic de pubalgie traités } \\
\text { de manière conservative } \\
95 \% \text { hommes } \\
\text { Moyenne d'âge : } 27.3 \text { (standard deviation (SD) } \\
4.6 \text {, range } 18-43 \text { years) } \\
\text { Sports identifiés dans les études : football (61\% of the } \\
\text { studies). Autres sports : hockey sur glace (7\%), football } \\
\text { australien (6\%) et rugby (6\%). (14\% sport pas précisé) } \\
\text { Durée moyenne des symptômes: } 10.9 \text { months } \\
\text { (SD } 4.7, \text { range } 3-21 \text { months). }\end{array}$ \\
\hline Pathologie(s) & $\begin{array}{l}\text { Diagnostiques de pubalgies } \\
\text { SANS : } \\
\text { Intra-articular hip pathologies (osteoarthritis and } \\
\text { femoroacetabular impingement) and isolated } \\
\text { nerve injuries }\end{array}$ \\
\hline Intervention & $\begin{array}{l}\text { Thérapie passive et/ou thérapie active par } \\
\text { l'exercice }\end{array}$ \\
\hline Nombre de sujets & 219 \\
\hline Groupe contrôle & $\begin{array}{l}\text { Thérapie passive ou thérapie active par } \\
\text { l'exercice }\end{array}$ \\
\hline Issues & $\begin{array}{l}\text { - Succès du traitement } \\
\text { - Taux de récupération } \\
\text { - Pourcentage d'athlètes de retour au sport } \\
\text { après le traitement } \\
\text { - Temps jusqu'au retour au sport } \\
\text { - Scores de douleur } \\
\text { - Scores fonctionnels } \\
\text { - Satisfaction du patient }\end{array}$ \\
\hline Suivi & $\begin{array}{l}\text { Moyenne de la durée du follow-up : } 27.7 \text { months } \\
\text { (SD 32.1) }\end{array}$ \\
\hline $\begin{array}{l}\text { Une population } \\
\text { importante a-t-elle été } \\
\text { exclue? }\end{array}$ & Sportifs autres que les footballeurs \\
\hline
\end{tabular}




\begin{tabular}{|l|l|}
\hline $\begin{array}{l}\text { Manques identifiés/ ce } \\
\text { qu'il reste à faire }\end{array}$ & $\begin{array}{l}\text { Les études futures devraient être attentifs aux } \\
\text { éléments suivants : manque de groupe contrôle, } \\
\text { randomisation, ... }\end{array}$ \\
\hline
\end{tabular}

Tableau 8 : Extraction des données de Serner et al. (2015)

\section{Annexe V : Qualité en fonction des points AMSTAR}

\begin{tabular}{|l|l}
\hline Points AMSTAR & Qualité de l'étude \\
\hline $10-11$ & Excellente qualité \\
\hline $8-10$ & Bonne qualité \\
\hline $6-8$ & Qualité moyenne \\
\hline $3-6$ & Mauvaise qualité \\
\hline $0-3$ & Très mauvaise qualité \\
\hline
\end{tabular}

Tableau 9: Qualité en fonction des points AMSTAR (Barras et Lutz, 2017) 